\title{
Analysis of Brain-Neuromuscular Synchronization and Coupling Strength in Muscular Dystrophy after NPT Treatment
}

\author{
Elio Conte ${ }^{1,2}$, Ken Ware ${ }^{4}$, Riccardo Marvulli1,3, Giancarlo Ianieri'3 ${ }^{3}$ Marisa Megna ${ }^{3}$, \\ Sergio Conte ${ }^{1}$, Leonardo Mendolicchio ${ }^{1}$, Enrico Pierangeli ${ }^{1}$ \\ ${ }^{1}$ School of Advanced International Studies on Applied Theoretical and Non Linear Methodologies of Physics, \\ Bari, Italy \\ ${ }^{2}$ Department of Basic Sciences, Neuroscience and Sense Organs, University of Bari Aldo Moro, Bari, Italy \\ ${ }^{3}$ Department of Physical Medicine and Rehabilitation, University of Bari Aldo Moro, Bari, Italy \\ ${ }^{4}$ The International NeuroPhysics Functional Performance Institute, Robina, Australia \\ Email: elio.conte@fastwebnet.it
}

Received 13 June 2015; accepted 2 August 2015; published 6 August 2015

Copyright (C) 2015 by authors and Scientific Research Publishing Inc.

This work is licensed under the Creative Commons Attribution International License (CC BY).

http://creativecommons.org/licenses/by/4.0/

(c) () D Den Access

\begin{abstract}
We perform an analysis of brain-neuromuscular synchronization and coupling strength in muscular dystrophy before and after NPT treatment. In order to estimate with accuracy the level of brain synchronization, we introduce and use the method of the cross GMI that was elaborated by Pompe. The finality is to account for the nonlinear chaotic dynamic contributions that regulate the dynamics. We find that this method is excellent and it may be applied in such case of SEMG and EEG study as well as at a general neurological level. By it we arrive to quantify the coupling strength and synchronization first between two muscular sections (left and right trapezes) and after between Brain activity, as recorded by the EEG, and the trapezes. We compare the results before and after the NPT treatment and we find that the improvement obtained following such treatment is very consistent. We have now in progress other studies relating in particular the application of such new methodologies to other more serious pathologies as the HSP (Hereditary Spastic Paraplegia).
\end{abstract}

\section{Keywords}

Chaos Analysis, Muscular Dystrophy, Brain Synchronization Analysis, Generalized Mutual Information, Surface Electromyography, EEG 


\section{Introduction}

In this year we have performed an intense activity of research focalized on the objective to analyze recorded data of subjects having muscular dystrophy. This activity has culminated with the publication of a number of papers on WJNS (World Journal of Neuroscience) and on WJCD (World Journal of Cardiovascular Diseases). In the first paper [1] we studied the dynamics of the SMR band in the EEG. This subject was subjected to what we have called Ken Ware Neuro Physics Treatment (NPT) and we used the nonlinear method of the Generalized Mutual Information (GMI) to analyze in detail the SMR band at rest and during the treatment. Generally speaking, brain dynamics responds to a chaotic-deterministic regime with a complex behaviour that constantly performs transitions of states self-rearranging and self-organizing its patterns. We demonstrated that the SMR chaotic dynamics responds directly to such regime and that also decreasing in EEG during muscular activity really increases its ability of self-arrangement and self-organization in brain. The proposed novel method of the GMI was arranged by us so that it may be used in several cases of clinical interest. In the case of muscular dystrophy examined, GMI enabled us to quantify with accuracy the improvement that the subject realized during the NPT treatment.

In the second paper [2] we devoted our efforts to autonomic neuroscience. As said, in the previous paper, we studied facioscapulohumeral muscular dystrophy before and after NPT treatment (NPT) using the nonlinear methodology of the Generalized Mutual Information (GMI) analysis of Sensory Motor Rhythm, and we produced detailed results evidencing that the mentioned NPT treatment involved a net improvement of the patient under his subjective psychological condition, and in particular, under the neurological and sensory motor profile. We quantified with accuracy the improvement that the subject realized during such treatment. Of course, previous studies of several authors (see in detail references in [2]) evidenced that muscular dystrophies are strongly linked to a profound ANS dysfunction. Therefore, the aim of the second study was to analyze the ANS of the subject before and after the treatment. We performed analysis in time as well as in frequency domain and by using nonlinear methods. The basic result of the paper was that, according to our analysis, the subjects started with a serious ANS dysfunction before the NPT treatment and that a net improvement was obtained after this therapy. All the examined parameters resulted strongly altered before the treatment and all they returned in the normal range after the NPT. Again we attempted on one hand to outline the advantage of the NPT, and on the other hand to use linear and nonlinear methods that may be used not only in the case of muscular dystrophy, but also, generally speaking, under a more generalized clinical profile in this field.

In the third paper [3] we discussed muscular dystrophy with performed HRV (Hear Rate Variability) analysis. The results were focalized on a subject evidencing a net ANS dysfunction before the NPT treatment. All the basic parameters relating standard time and frequency domain of HRV analysis resulted profoundly altered. Examination by Poicaré plot evidenced in particular that the subject had an atrial fibrillation. Nonlinear indexes ApEn and Samp En evidenced the very high condition of risk of the subject. The emerging indication was for the cardiologists and scholars in cardiovascular disease with the direct suggestion to perform always ANS investigation in subjects in case of muscular dystrophy.

In the fourth paper [4], we analyzed muscular dystrophy recorded, as in all our previous cases, by sEMG and we used the standard methodologies of sEMG analysis and advanced nonlinear chaotic methods here including the RQA (Recurrence Quantification Analysis). We reached evidence that the sEMG signal contains a large chaotic component. We estimated the correlation dimension (fractal measure), the largest Lyapunov exponent, the LZ complexity and the \%Rec and \%Det of the RQA demonstrating that such indexes of RQA are able to detect frequent chaotic transitions as well as the presence of repetitive hidden patterns in sEMG, which in turn, senses the level of MU synchronization within the muscle. The results gave a totally new and interesting methodological plan in the sense that they pointed out the manner in which nonlinear methods and RQA must be arranged and applied in clinical routine in order to obtain results of clinical interest. Under the direct clinical profile, we studied, as said, the muscular dystrophy and evidenced that the continuous regime of chaotic transitions that we have in muscular mechanisms may benefit in this pathology by the use of the NPT treatment.

As seen, we have developed a large body of research and we have had two finalities. The first one has been to investigate such NPT treatment in detail. The contents of this treatment have been exposed by us in the course of our previous papers and consequently we will not expose it here again in detail. The conclusion is that this NPT method actually induces a net improvement. We have examined in detail not only muscular dystrophy but also other more serious pathologies as HSP (Hereditary Spastic Paraplegia) and our emerging indication is that it 
gives very encouraging results.

The second pursued objective has been that one to give methodological new indications. In our analysis we have always used standard methodologies and new advanced methods all based on nonlinear methodologies evidencing that we are in presence of nonlinear-chaotic continuous transitions in sEMG analyzed mechanisms that require such advanced methods to finally obtain also new quantified indexes of very important clinical interest. Consequently we conclude that our new approach deserves to be employed from scholars that are active in such areas of investigation and clinical application.

The aim of the present paper is to complete the exposition of the results of our research on muscular dystrophy.

In fact, we have to explore still the argument of the synchronization and coupling strength brain-MU before and after the NPT treatment. It may be easily understood that this kind of research represents the most important section of our work since we will arrive to examine the level of synchronization and coupling strength existing between brain and muscular level and thus at neuromuscular level. We will report here the obtained results confirming again our double finality: from one hand we indicate the importance of the NPT treatment and from the other hand we give new methodological profiles illustrating the method that in such case deserves to be used in order to obtain valuable clinical information.

\section{Materials and Methods}

We will not enter in the details of the employed signal monitoring and in the exposition of the clinical cases since we have examined and exposed in all the details the experimental background in our previous papers [1]-[4]. Also we will not expose the NPT treatment since it was discussed in detail in the same publications. Only we will re-outline here that we recorded sEMG at a frequency of $980 \mathrm{~Hz}$ in six different channels ch1-ch6 (and in particular ch1 and ch2 right and left trapezes, respectively), in ch7 we recorded simultaneously the ECG and in CH8 the EEG. For the analysis we used the method of the Generalized Mutual Information and, in this case, cross Generalized Mutual Information that were introduced by Pompe [5] and that we also discussed in detail in our previous publications [1]-[4].

\section{Analysis of Synchronization}

Previously in [1]-[4], we exposed in detail the importance to analyze MU synchronization in sEMG.

In the case of the sEMG studies we have to quantify synchronization of MUs in time in the same muscular region as well as we have to estimate synchronization and coupling between different regions as it is the case of ch1 and ch2. We know that we are considering neuromuscular processes that are regulated by the central nervous system. Consequently, it is important to arrive finally to quantify synchronization and coupling strength between ch1-2 (trapezes)-and ch8 (Brain).

Let us repeat here the general criteria that we used in our investigations:

Criterium a:

generally speaking, chaoticity increases at the beginning of the muscle task, as the result of further MUs recruitment, and then decreases in presence of fatigue phenomena and MUs synchronization.

Criterium b:

generally speaking, considering frequencies firing rate we have that for Fast Twich motor units (Phasic Type II) we have the frequency range 126 - $250 \mathrm{~Hz}$. For Tonic Type I Slow Twich motor units the range is 20 - $125 \mathrm{~Hz}$.

Let us sketch the situation. We have ch1 (right trapeze) and ch2 (left trapeze) signals, recorded as time series of data.

\subsection{Analysis of Synchronization and Coupling Strength between Right and Left Trapeze}

Following the method of the cross GMI (cross mutual information) we may examine synchronization and coupling strength between ch1 (right) and ch2 (left) trapeze at various time lags. When the estimated value of the cross GMI values, involving ch1 respect to ch2, evidence peaks, this means that we have here synchronizations and stronger coupling between the two considered time series and thus between the two recorded electrophysiological activities. By this way of investigation we obtain obviously information at neuromuscular level. Let us 
add some other general information.

We have to observe the dynamical interacting behaviours of two systems, and we have to establish how we can tell if they are coupled or not. A classical system is given as example in physiology observing the cardiorespiratory mechanism. Traditionally, the problem seems simple to be solved since we may start from the fact that, generally speaking, one can test to see if there is a degree of correlation, CF (Correlation Coefficient), between variables observed from each system. In fact, in the past in the traditional analysis in systems with many components, cross correlation, cross CF, in the time domain and cross spectrum or coherence in the frequency domain have the long been the mainstays of correlation detection. Unfortunately, however, cross correlation (cCF) measures only the linear correlation between two variables, and the cross spectrum is the Fourier transform of the cross correlation. Measures of cross correlation or coherency in effect ask whether there exists a functional relationship between the time series in question. However, the functional relation is albeit a very specific one for these linear measures. Nevertheless, for linear systems, these linear measurements are sufficient to describe the dynamical interdependence of their parts. Unfortunately, the components of complex extended systems in nature rarely display only a linear interdependence, and superposition may break down in describing their aggregate behaviour. Consider that we are examining a case of physiological interest where chaotic-deterministic mechanisms are acting and are represented instead from non linear, non stationary and non periodic dynamics of activity and control. Consequently, an appropriate methodology is required to study dependence and synchronization in such systems.

In the case when two arbitrary nonlinear systems are coupled, it is possible that their temporal evolutions become "synchronized" as one adjusts the coupling strength between them, even though their temporal evolutions will not be identical. The counterpart of such explanation is that in our case we have to study synchronization and coupling strength acknowledging that we are examining sEMG neuro muscular activity that of course is regulated also by a common driver that is at the neurological level (the brain control activity that we have recorded by EEG in ch8). Consequently we have to analyze dependency and synchronization between ch1 (right trapeze) and ch2 (left trapeze) accounting for non linear-chaotic contributions. This is to estimate dependency and synchronization between the two muscular regions at the different time lags $\tau$. In order to estimate the efficacy of the NPT treatment we have to perform such analysis at rest on sEMG signals obtained before and after the NPT treatment.

\subsection{Analysis of Synchronization and Coupling Strength between Brain and Right or Left Trapeze}

Soon after we have to consider directly the role of the neurological component, the common driver that we have previously mentioned. Consequently we have to maintain the basic theoretical foundations of the methods but changing this time the used time series. We will consider this time ch1, ch8, where ch8 represents this time the EEG (and thus the brain activity) that was recorded simultaneously to ch1 and ch2 in order to inspect in detail the synchronizing dynamics of the neurological component.

The reader will find that our analysis is divided in two sections. In Figure 1 we have the results of synchronization and analysis between right and left trapezes. Instead in Figure 2 and Figure 3 we have the results on synchronization and coupling strength at a proper neuromuscular level and thus invoking in the analysis the signal arising from the brain by the EEG. Let us see the results of Figure 1. We compare the obtained synchronization and coupling strength between ch1 and ch2 (right and left trapeze) before and after the treatment In each figure as well as in all the other following figures we have in ordinate the value of the dependency and thus of the synchronization and coupling strength while instead in abscissa we have the values of time delay. Each time delay value corresponds to 1.11 milliseconds. Each graph corresponds to about 400 milliseconds of observation. This is to say that in the twenty figures we observe step by step the behaviour of the synchronization and coupling strength examined step after step during the whole recording of ch1 and ch2 that we realized. Following the graphs, each time we may estimate the value of the dependency, the peaks, and the corresponding time delays and thus the appropriate frequencies of synchronization and coupling strength. Note that in time delays we have negative as well as positive values. Negative values indicate the milliseconds of anticipation (respectively of retardation) of ch1 respect to ch2 (and respectively (in the following figures) of ch1 respect to ch8 and ch2 respect to ch8). The typical time delays with peaks cover the range from 10 to 400 milliseconds. The peaked frequencies results in consequence in the range $125-10 \mathrm{~Hz}$. Looking at the figures we deduce that synchronization 


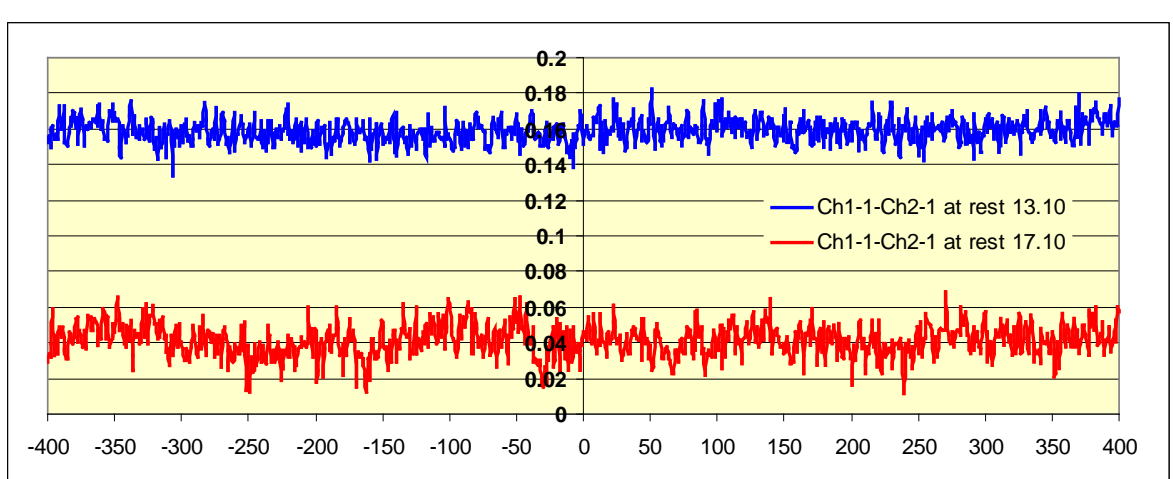

(a)

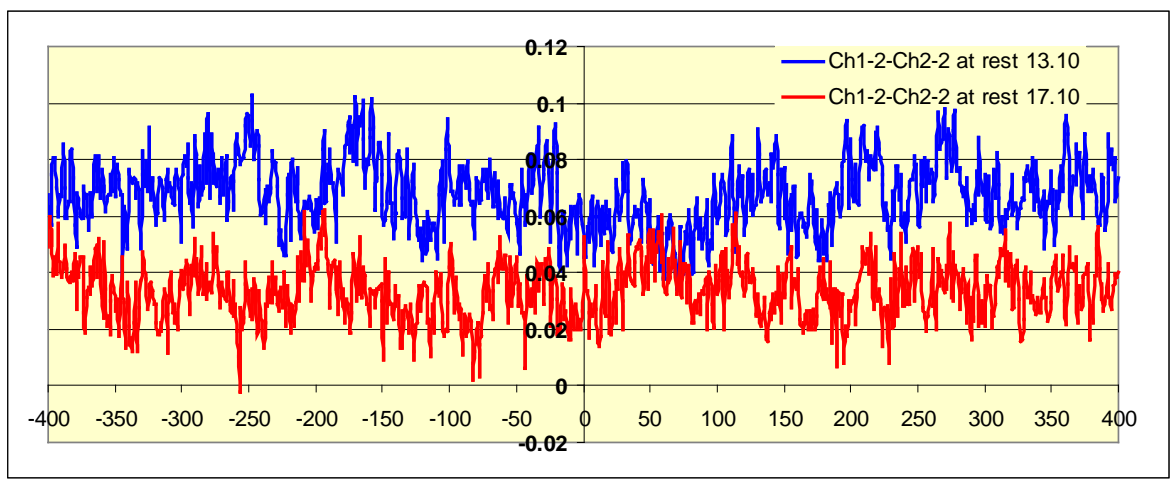

(b)

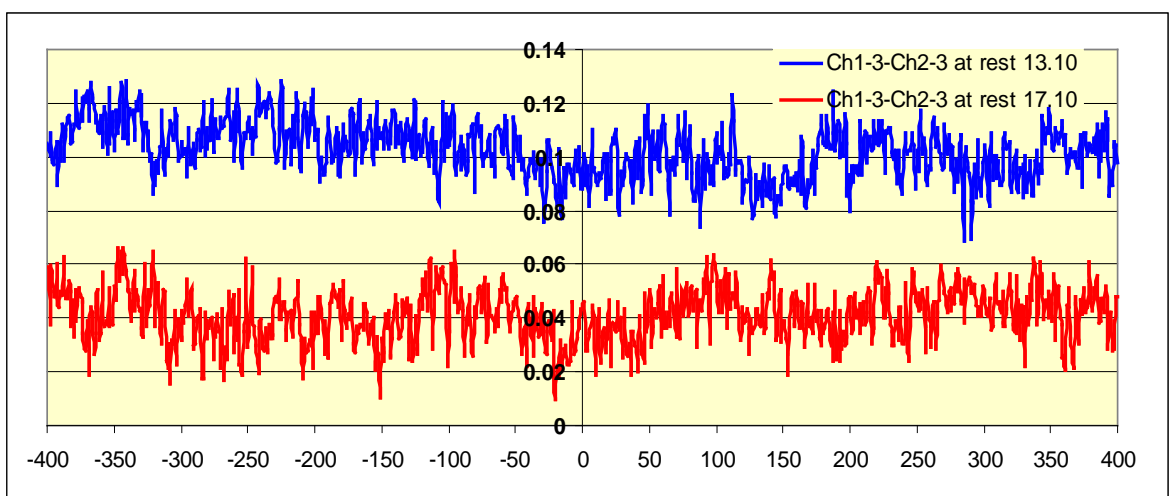

(c)

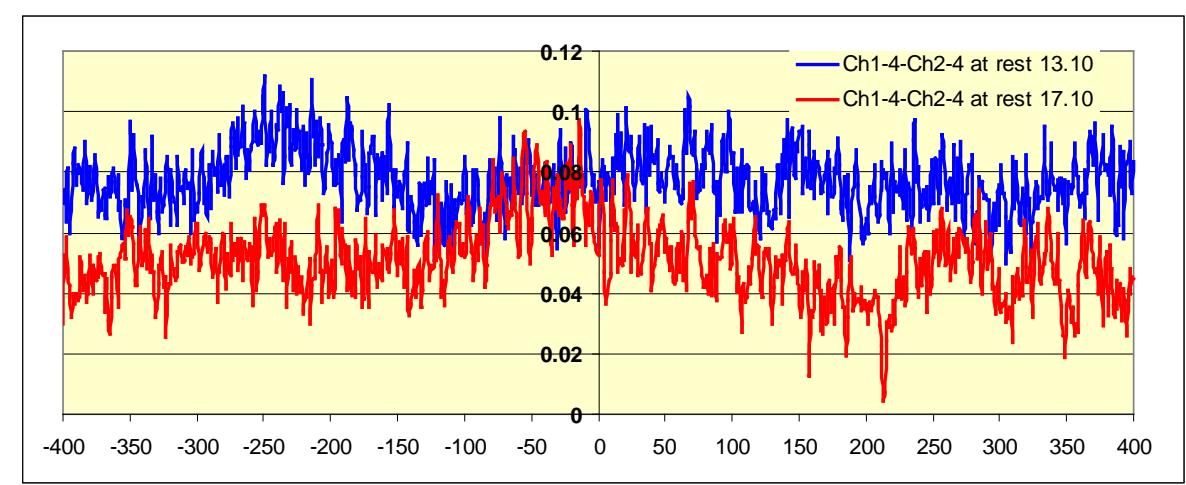

(d) 


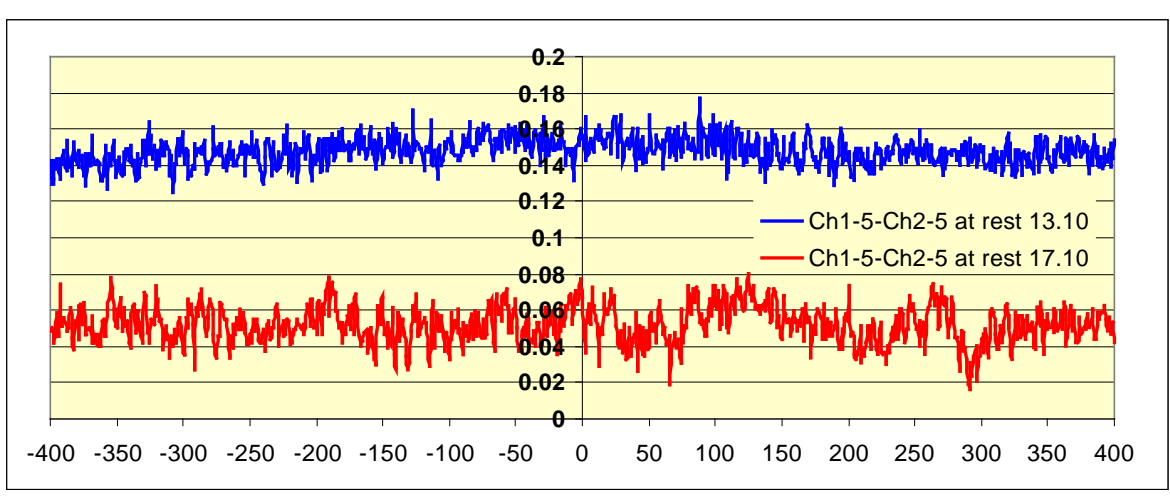

(e)

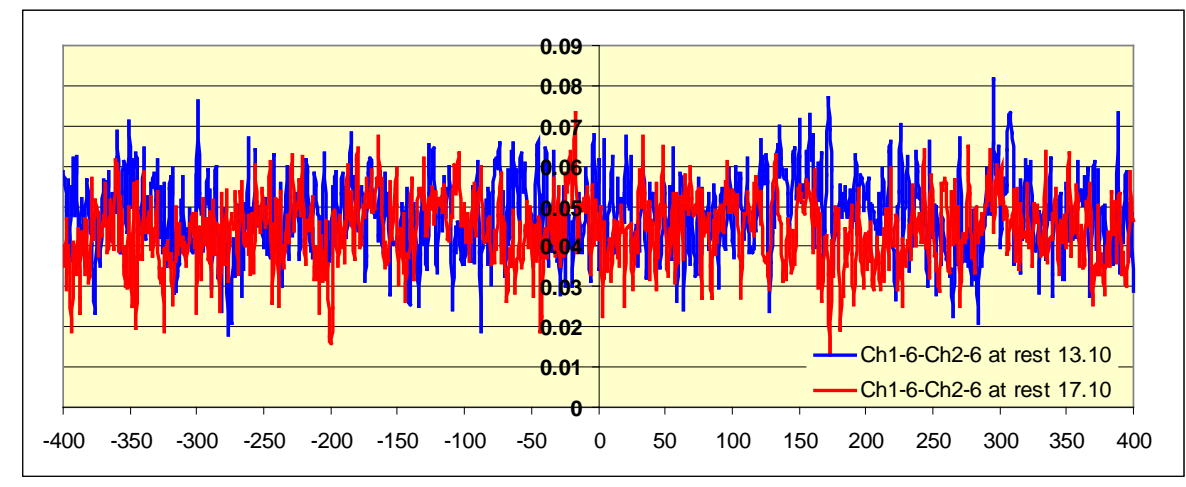

(f)

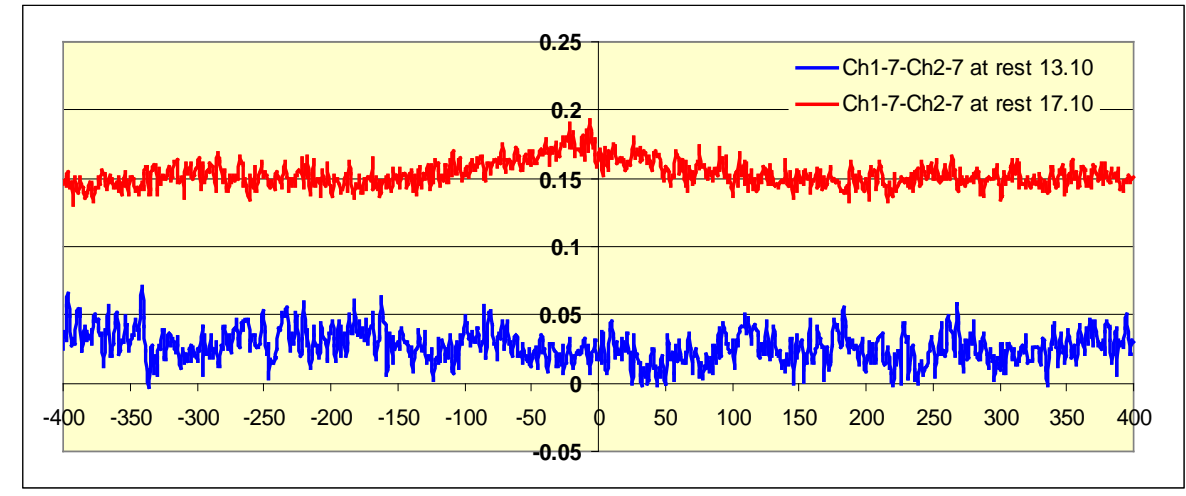

(g)

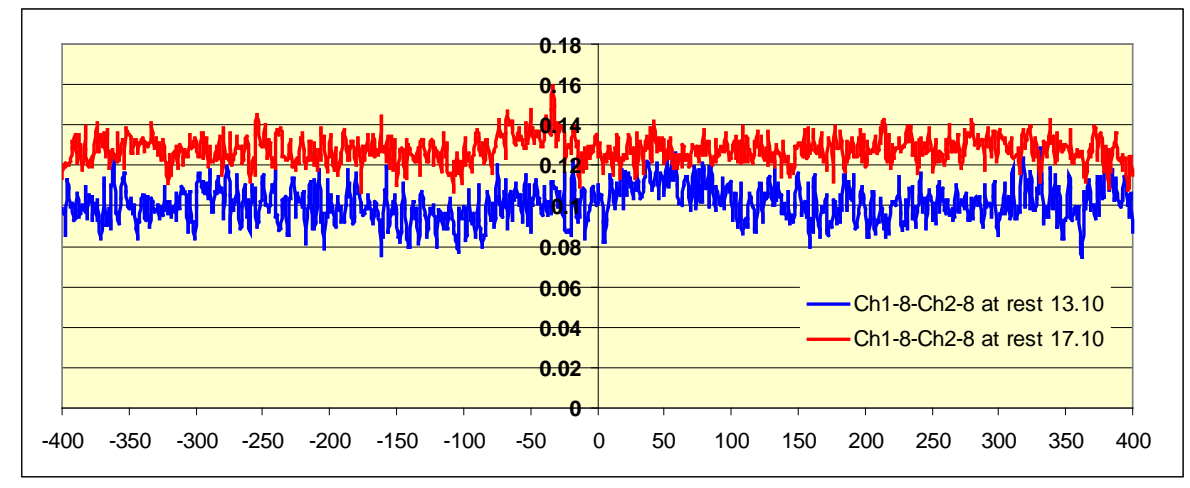

(h) 


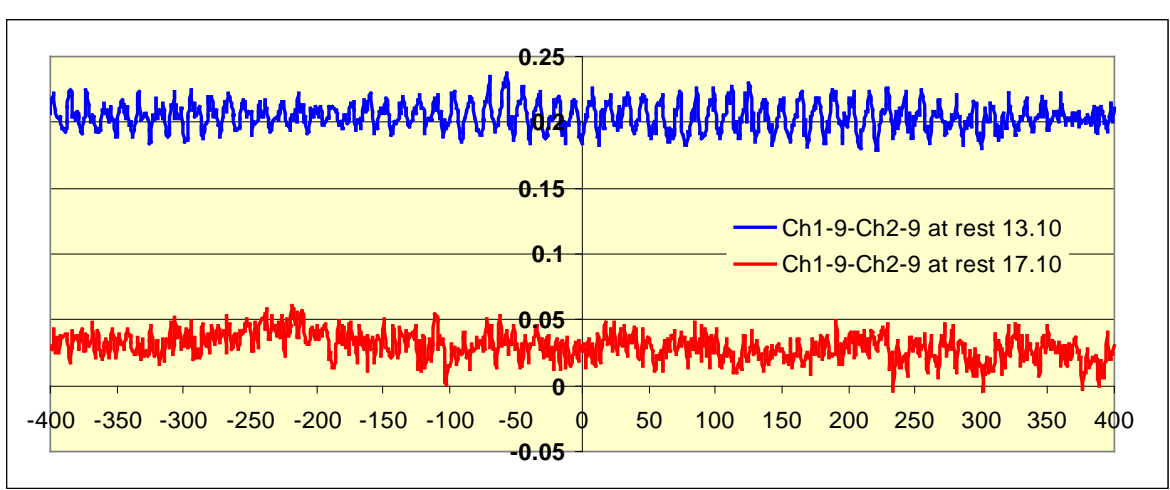

(i)

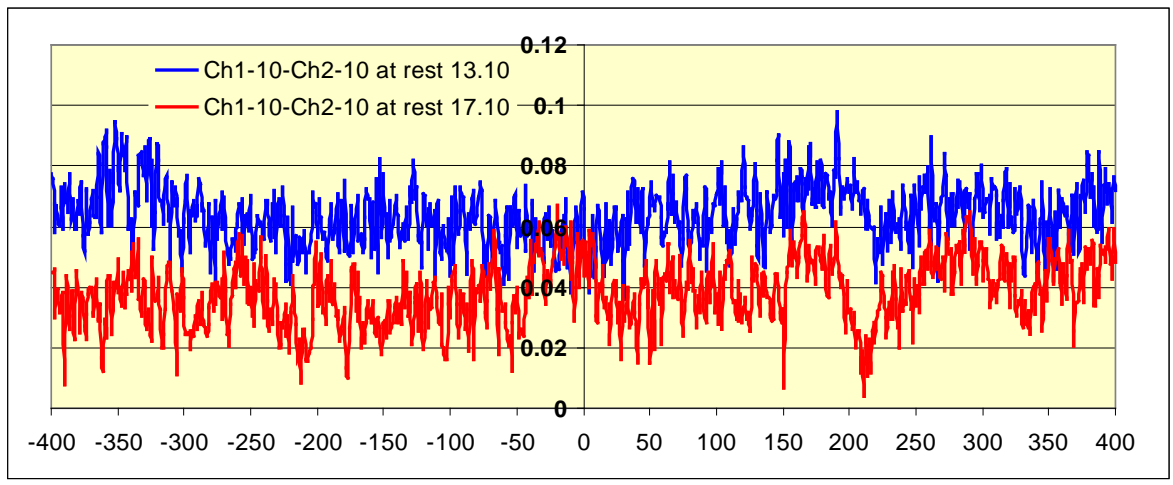

(j)

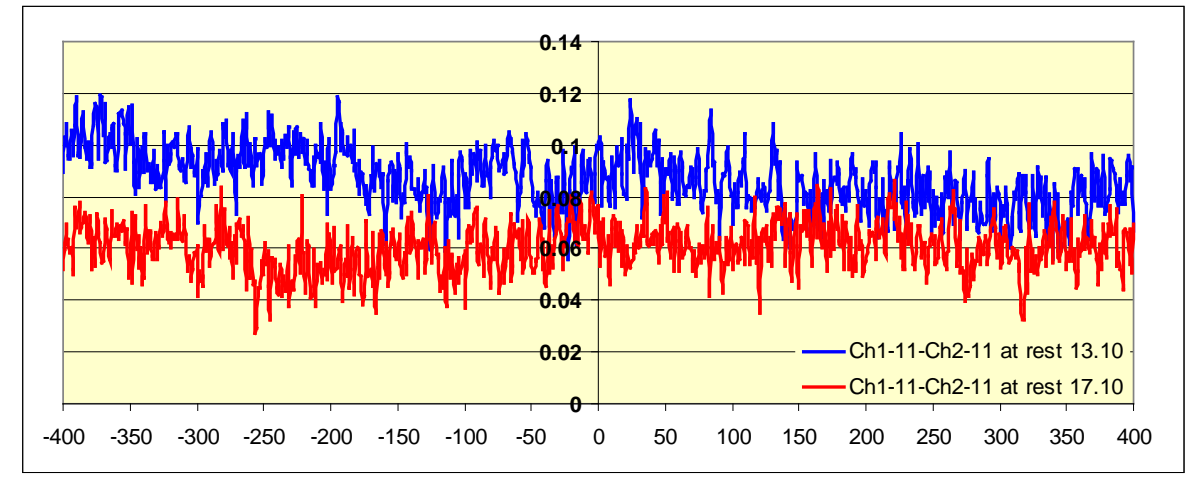

(k)

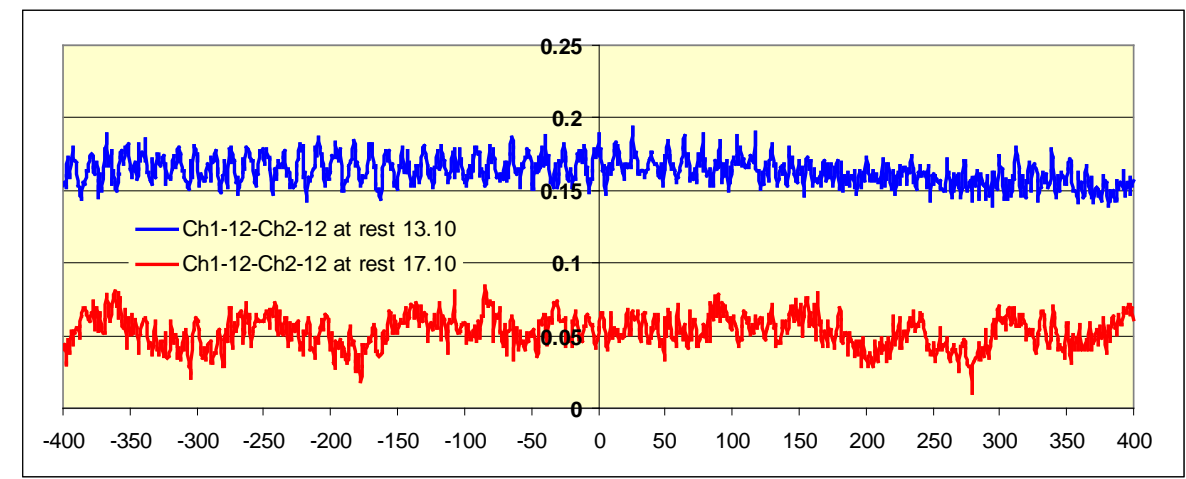

(l) 


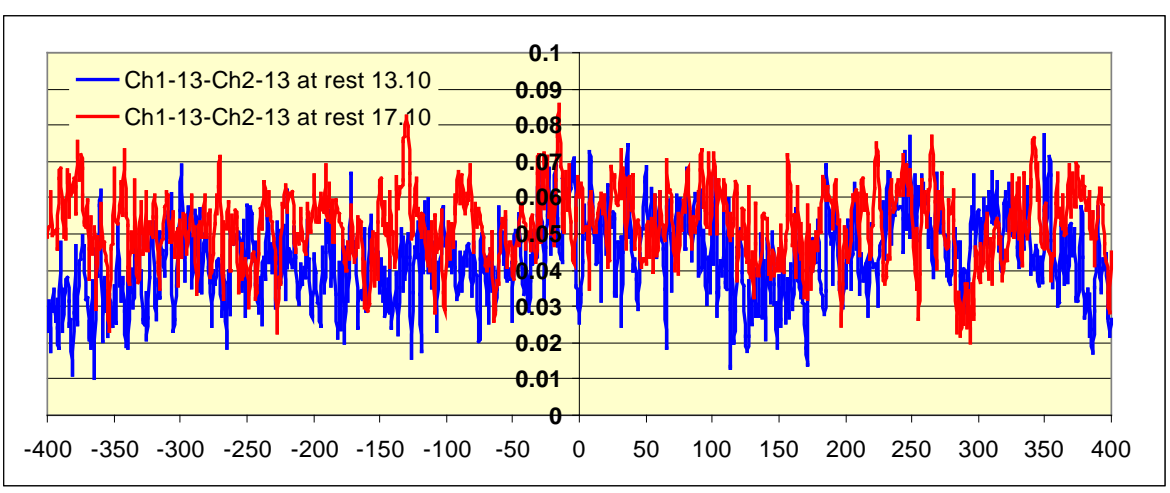

(m)

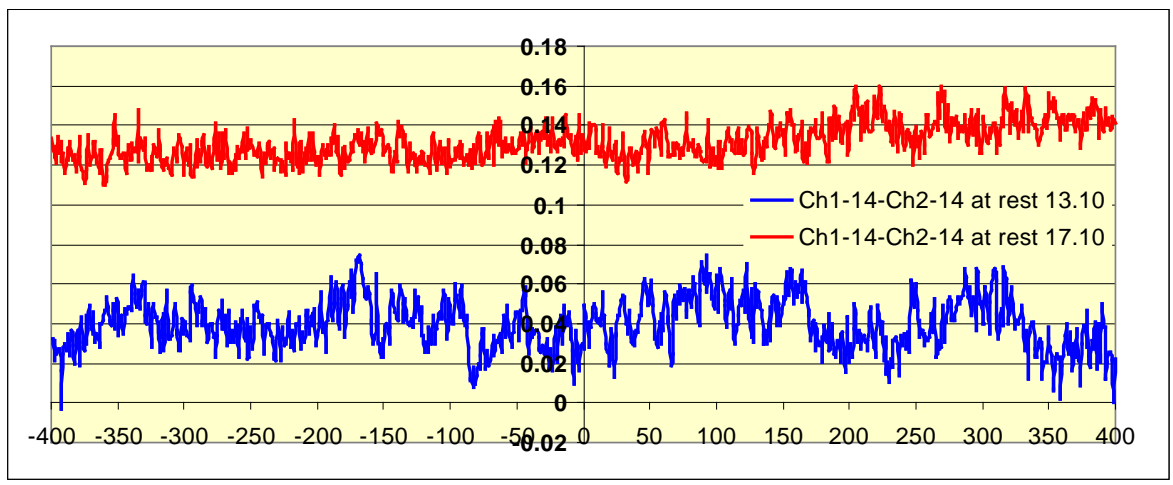

(n)

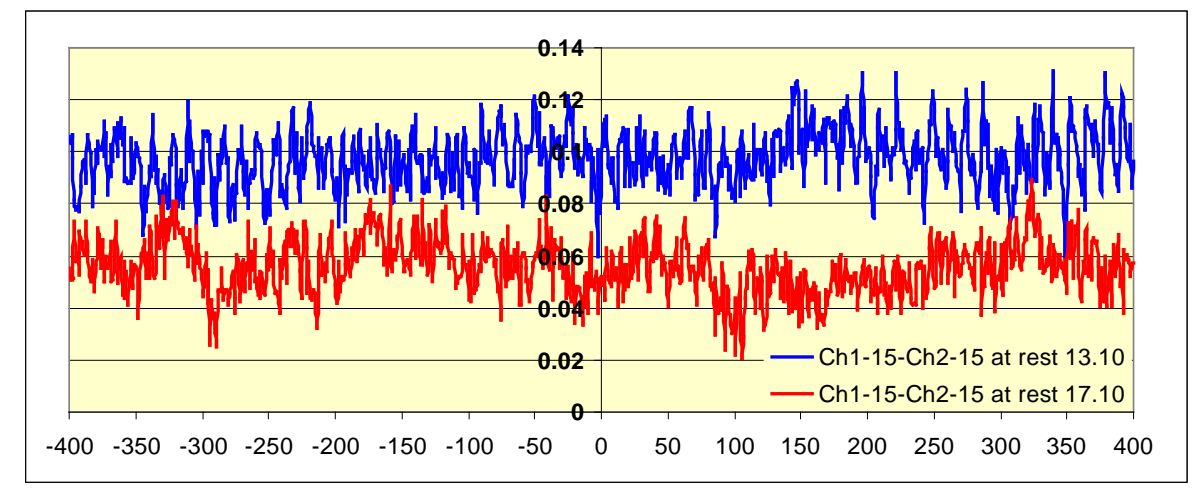

(o)

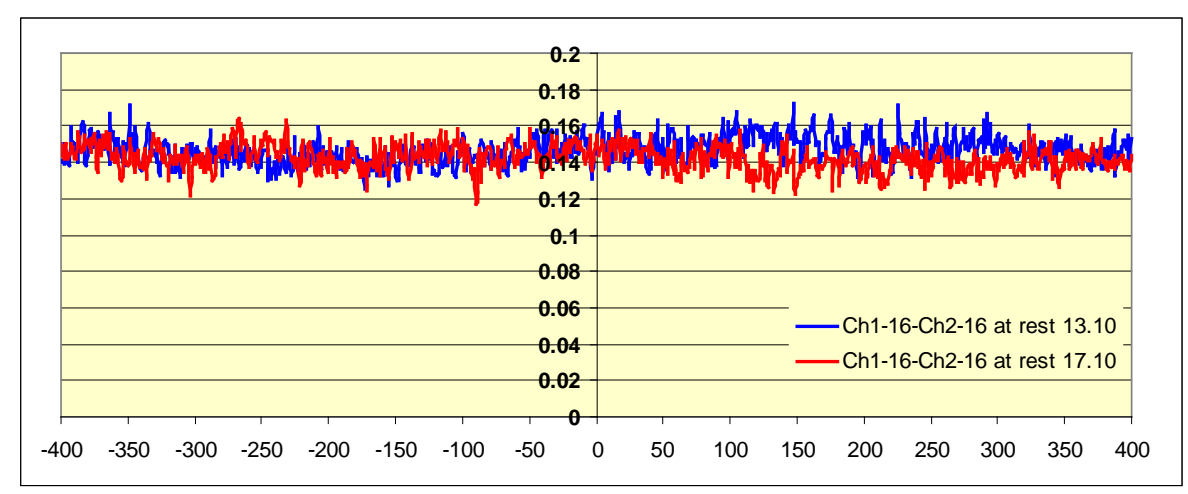

(p) 


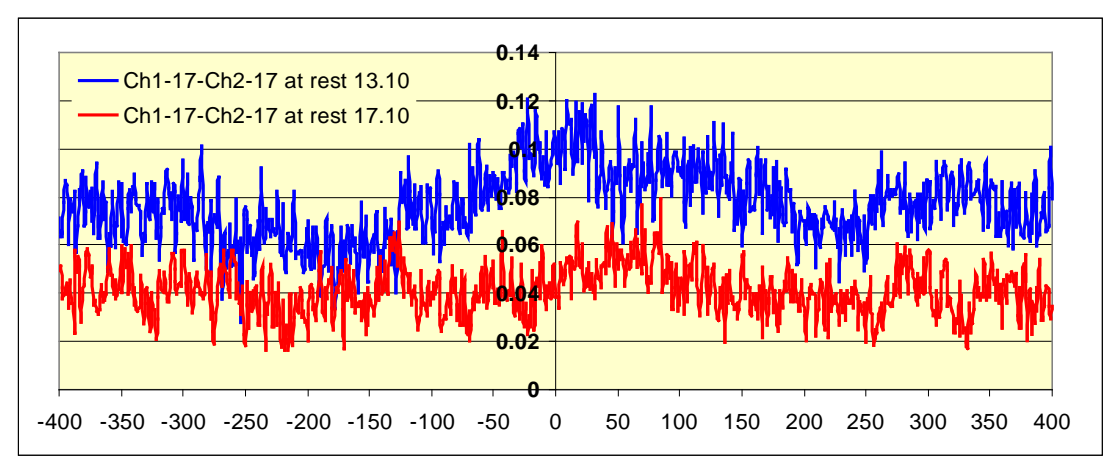

(q)

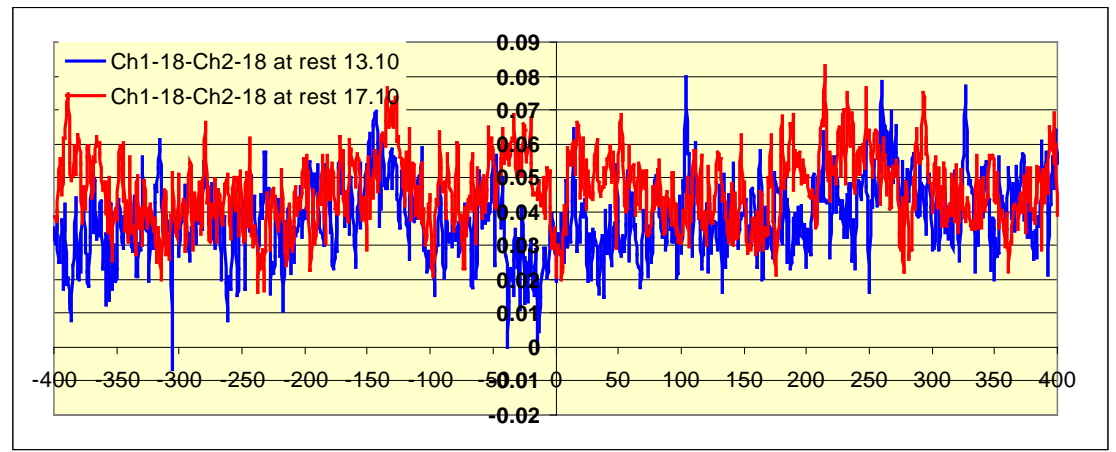

(r)

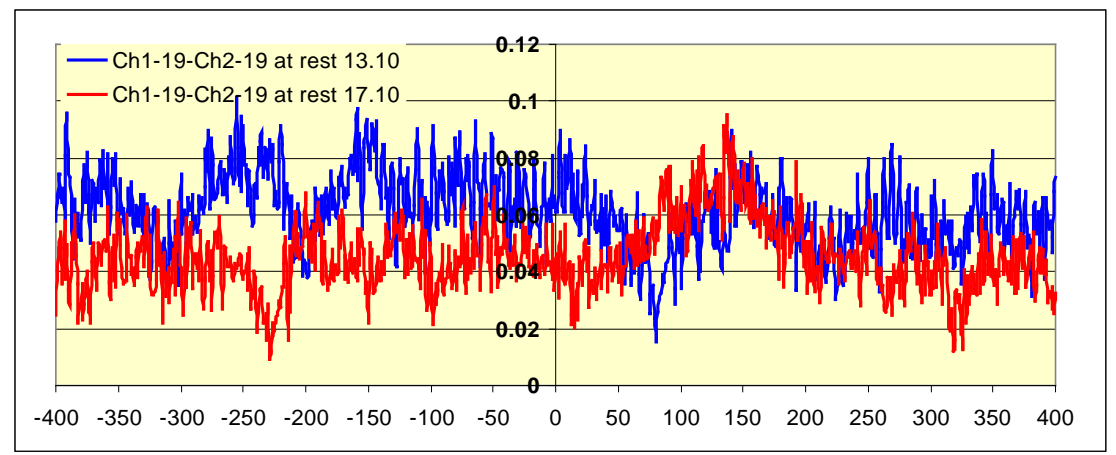

(s)

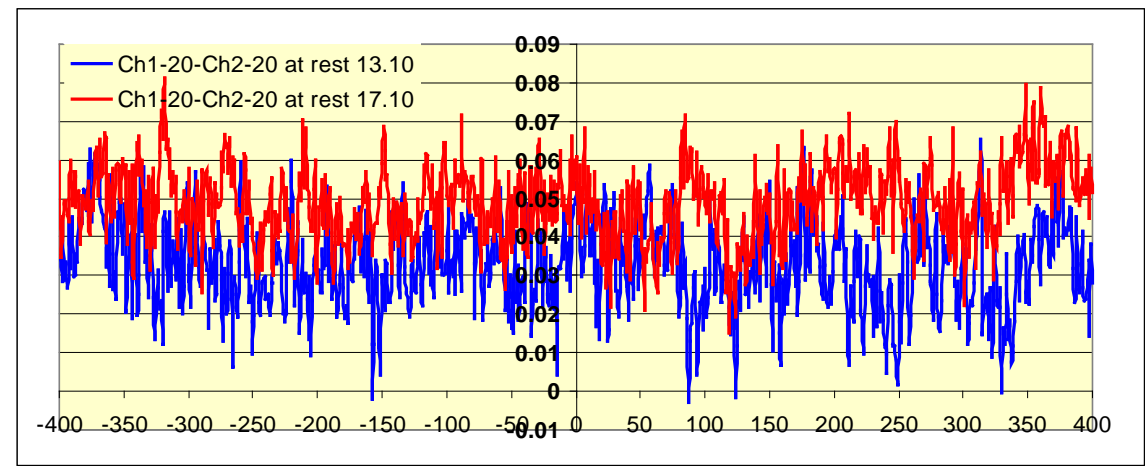

(t)

Figure 1. Examination of synchronization and coupling strength between ch1 (right trapeze) and ch2 (left trapeze) step by step. Each figure covers a range of time of about 400 milliseconds. Each time the behaviour is given before (13.10) and after (17.10) the NPT treatment. 


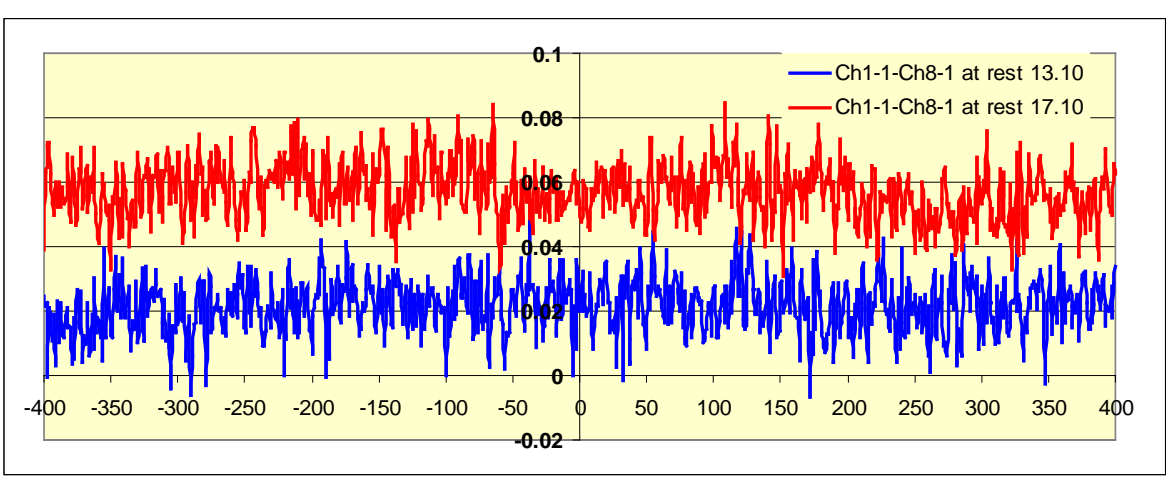

(a)

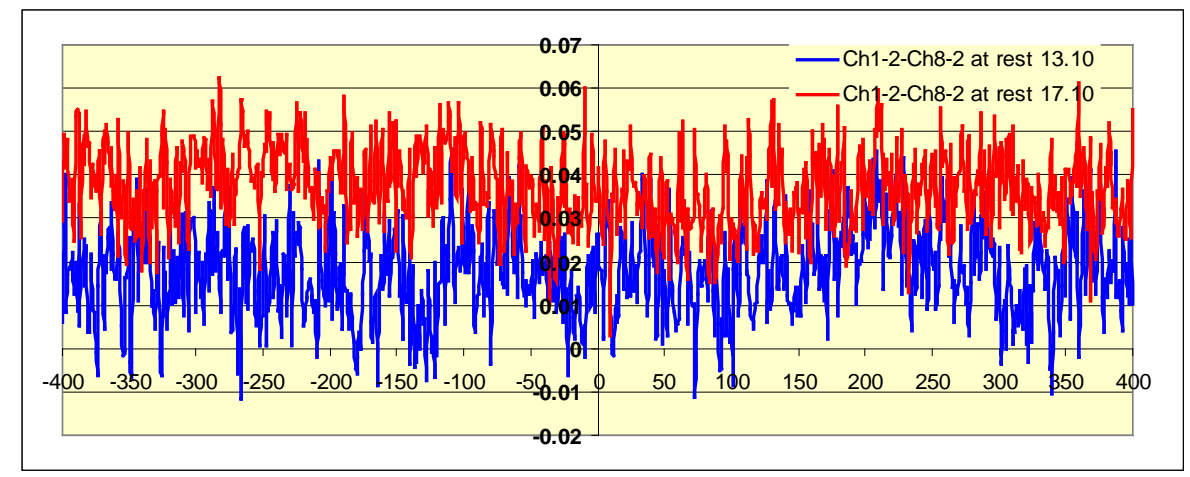

(b)

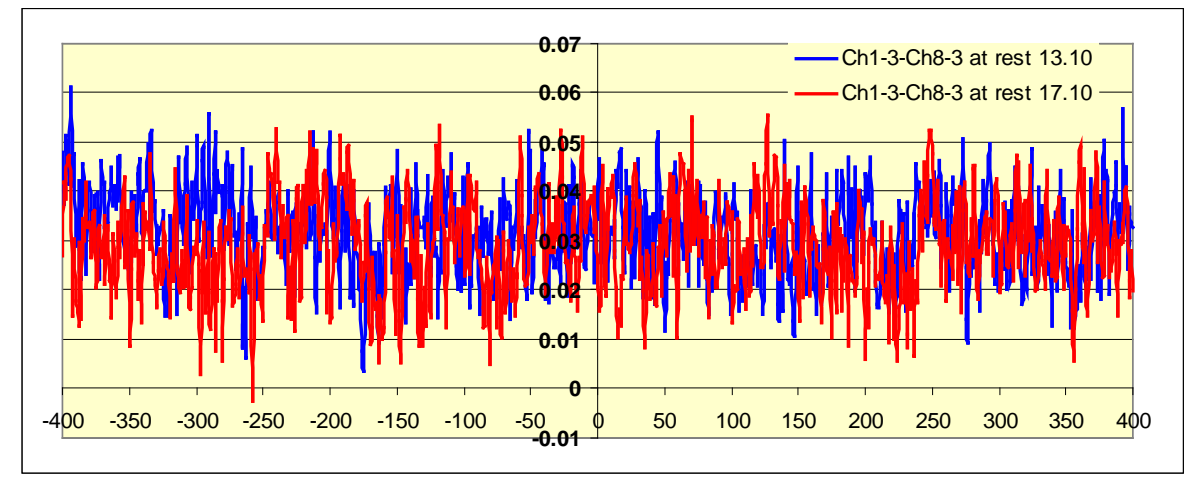

(c)

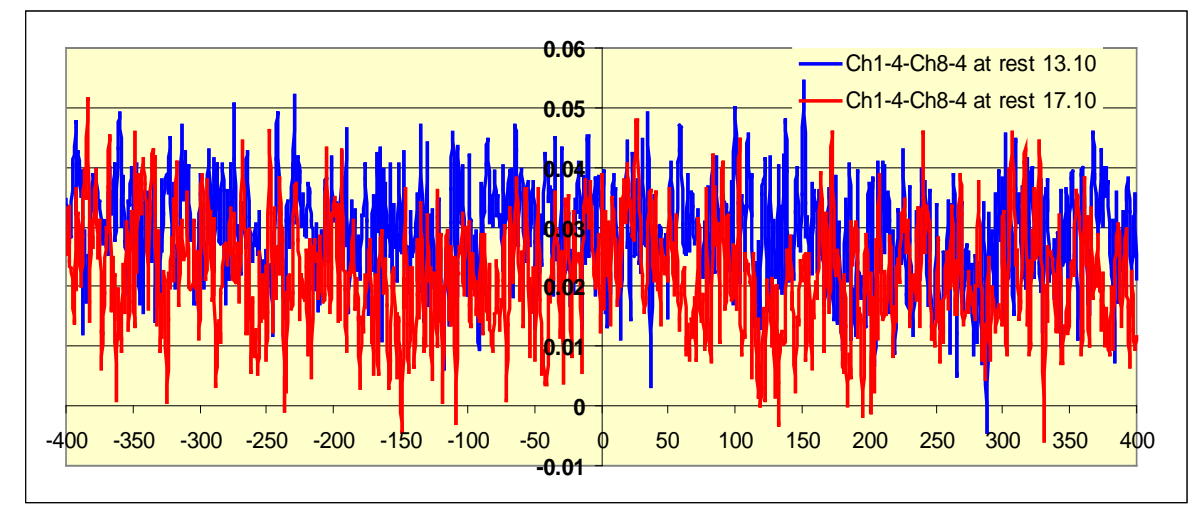

(d) 


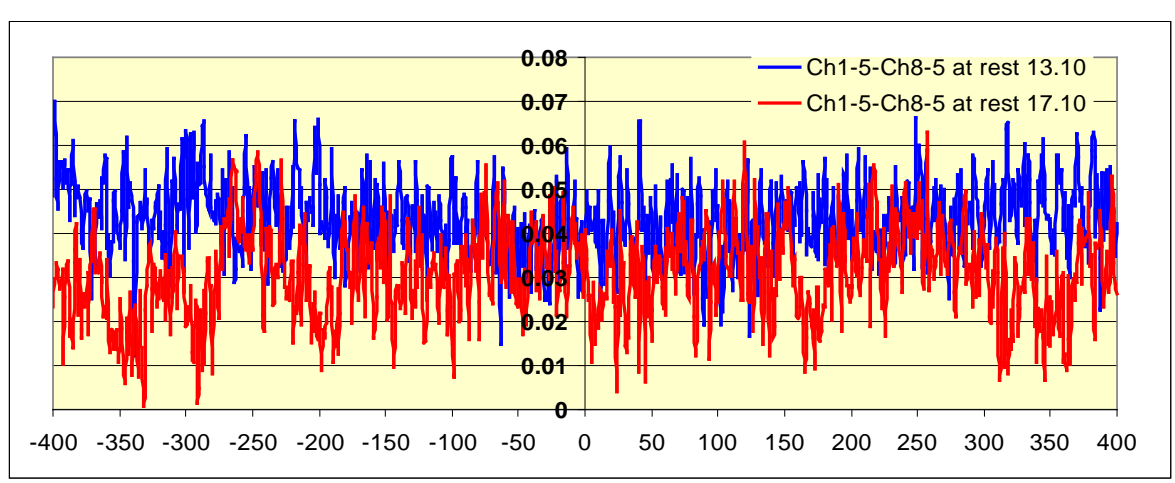

(e)

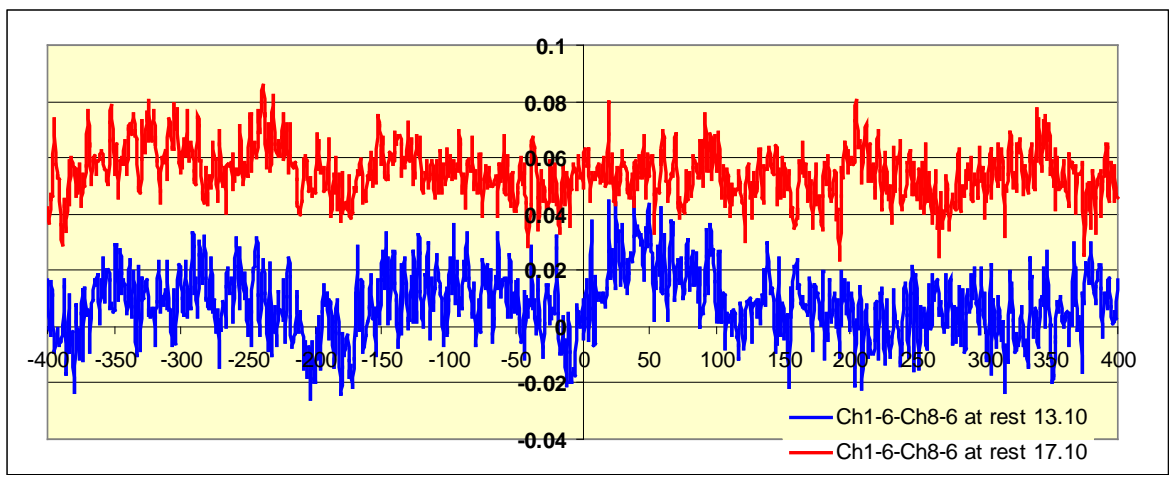

(f)

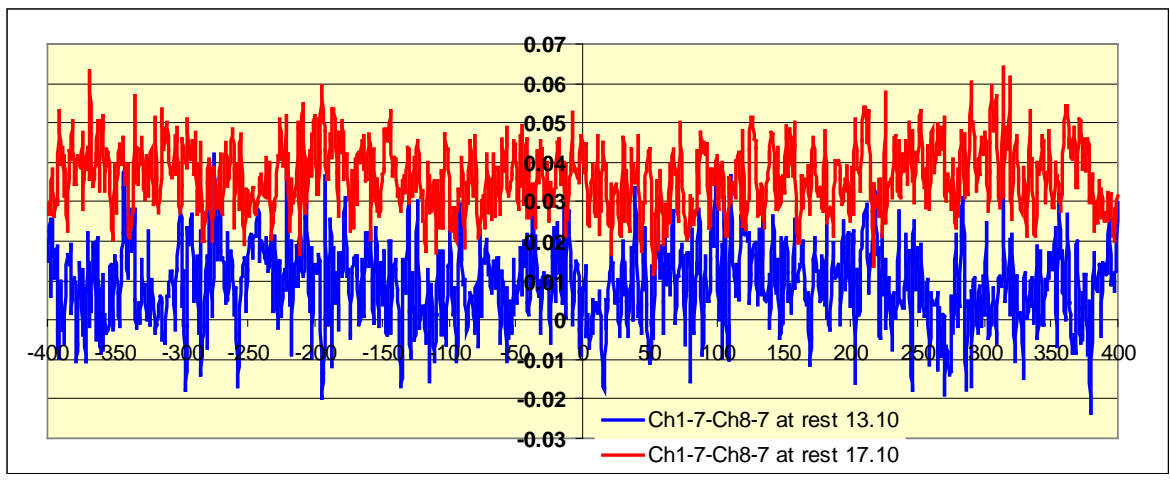

(g)

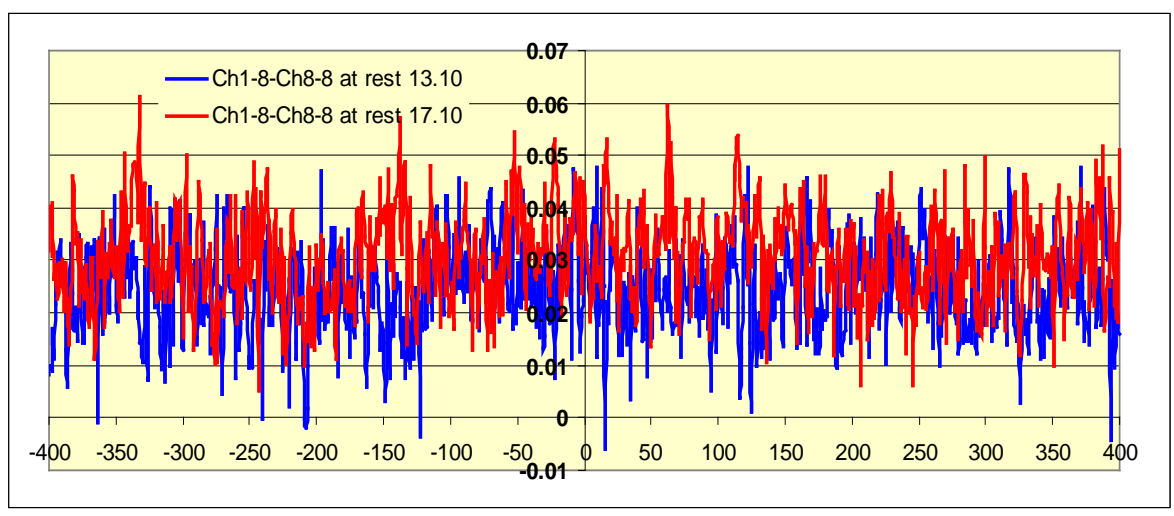

(h) 


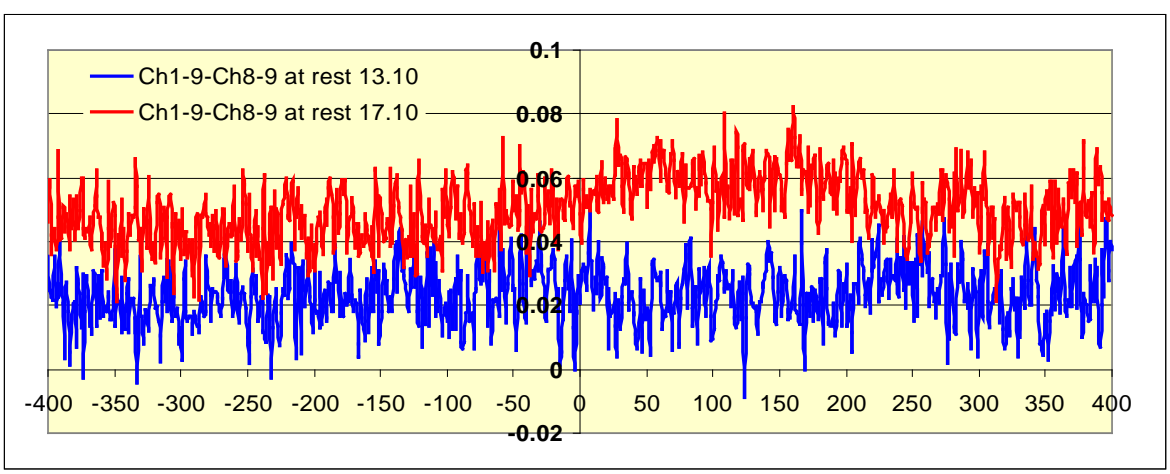

(i)

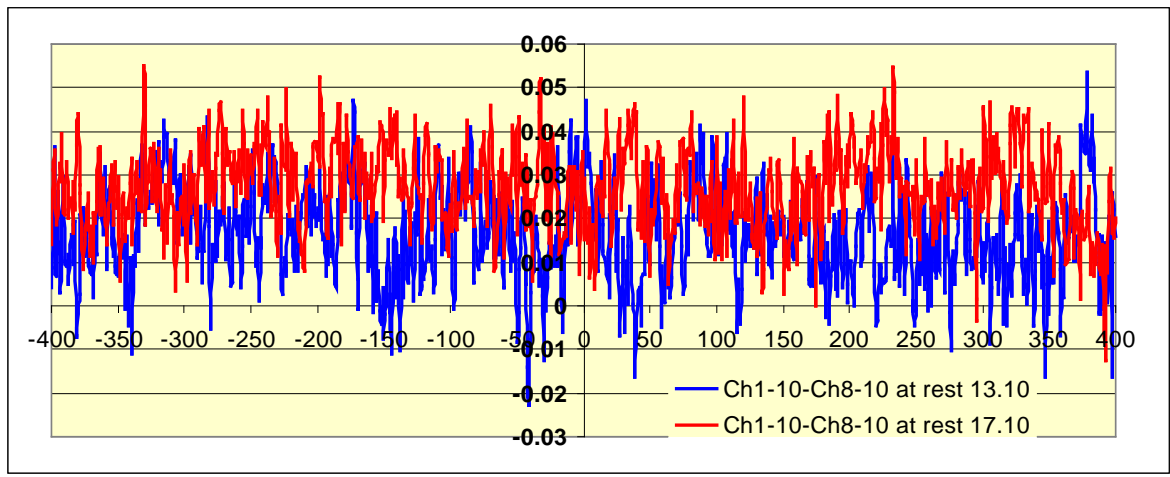

(j)

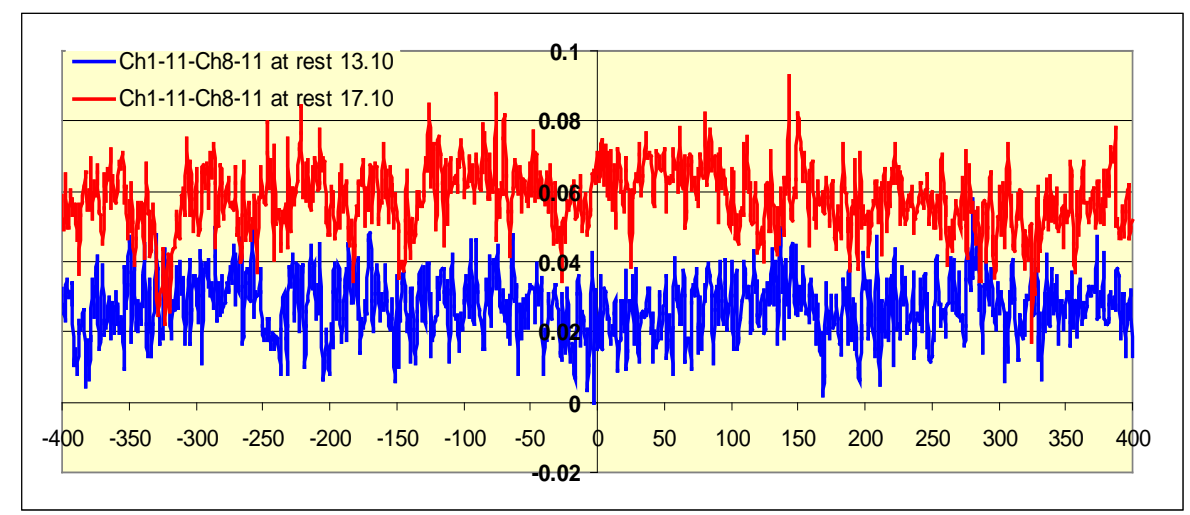

(k)

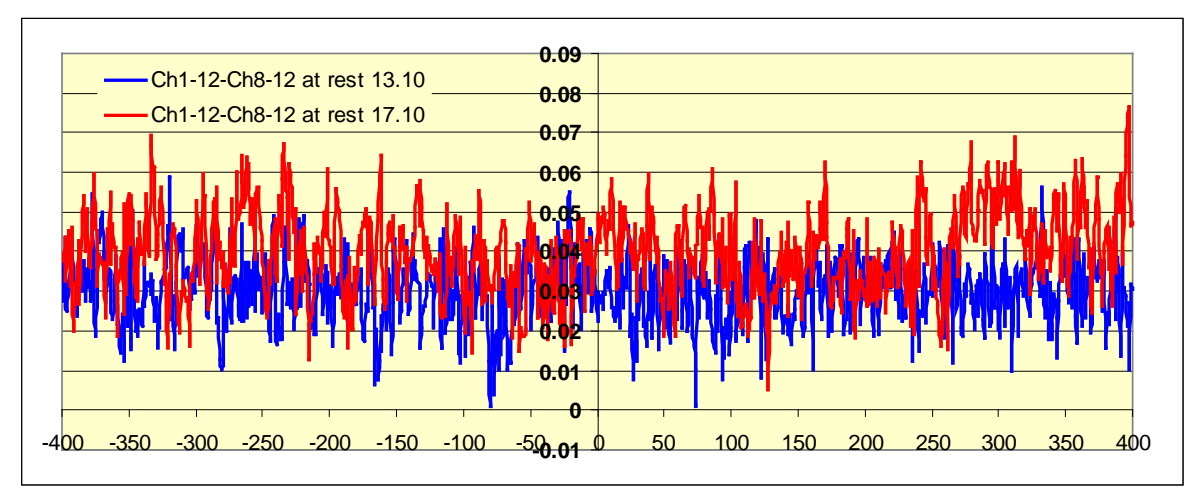

(l) 


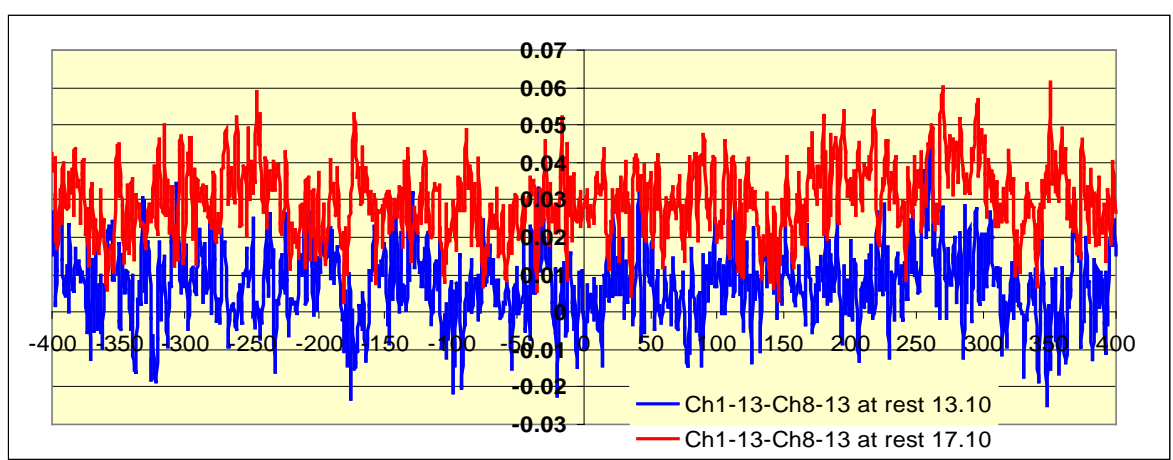

(m)

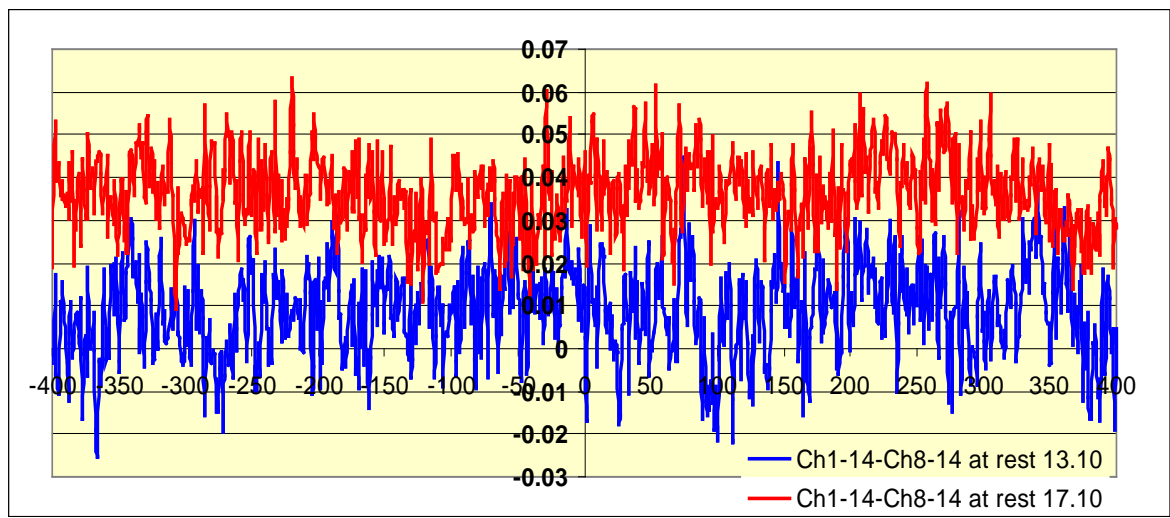

(n)

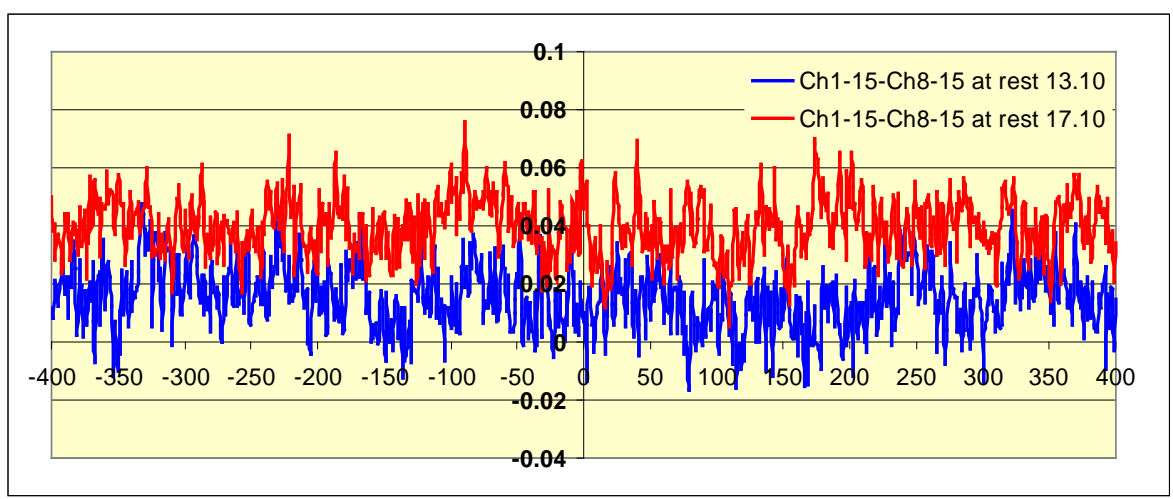

(o)

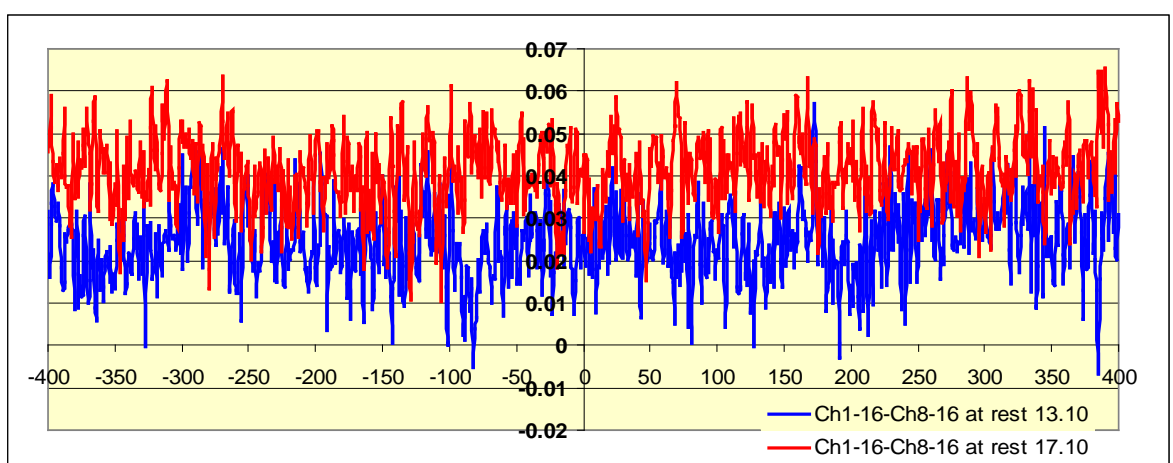

(p) 


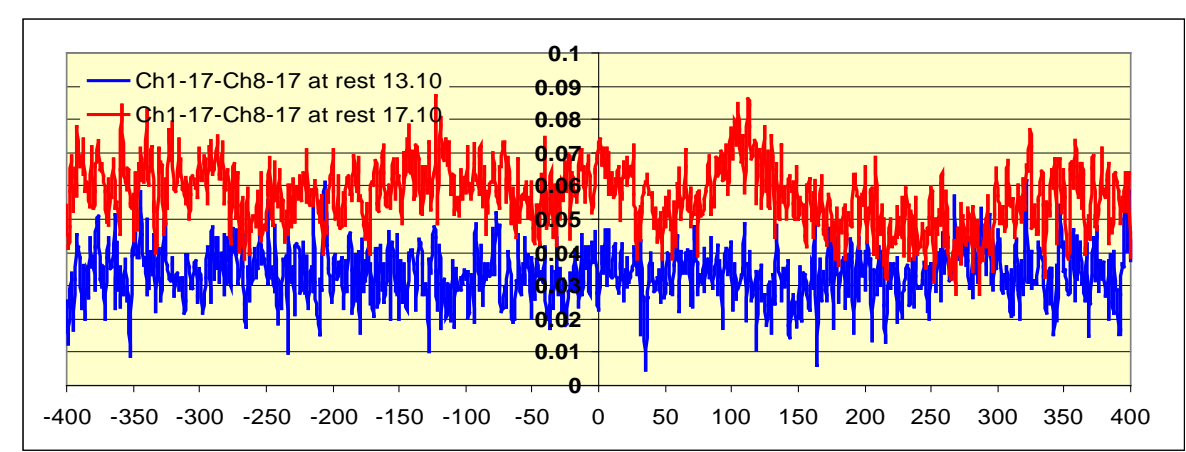

(q)

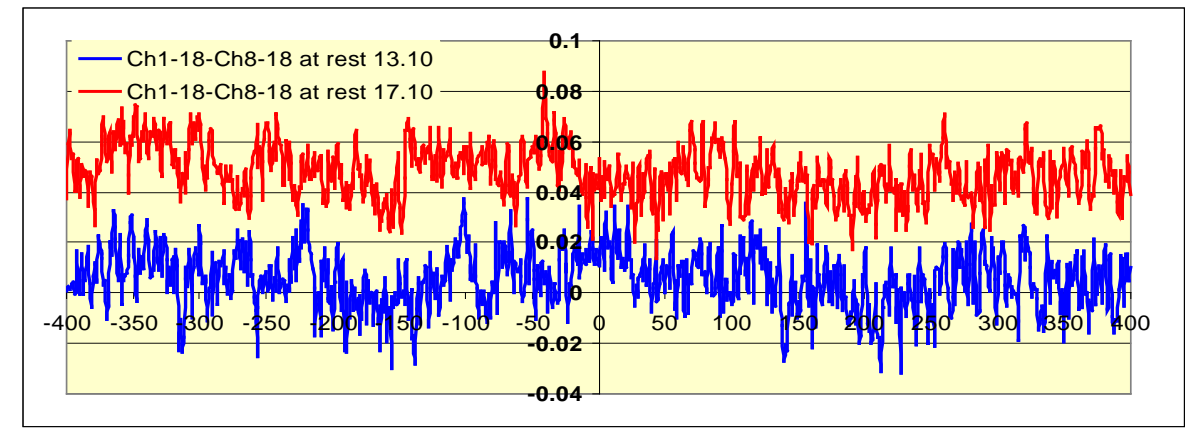

(r)

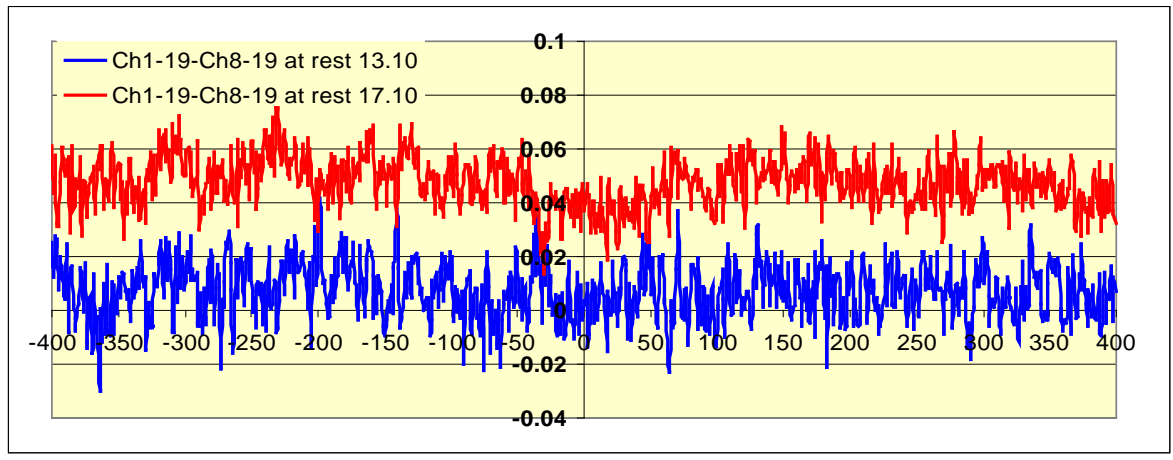

(s)

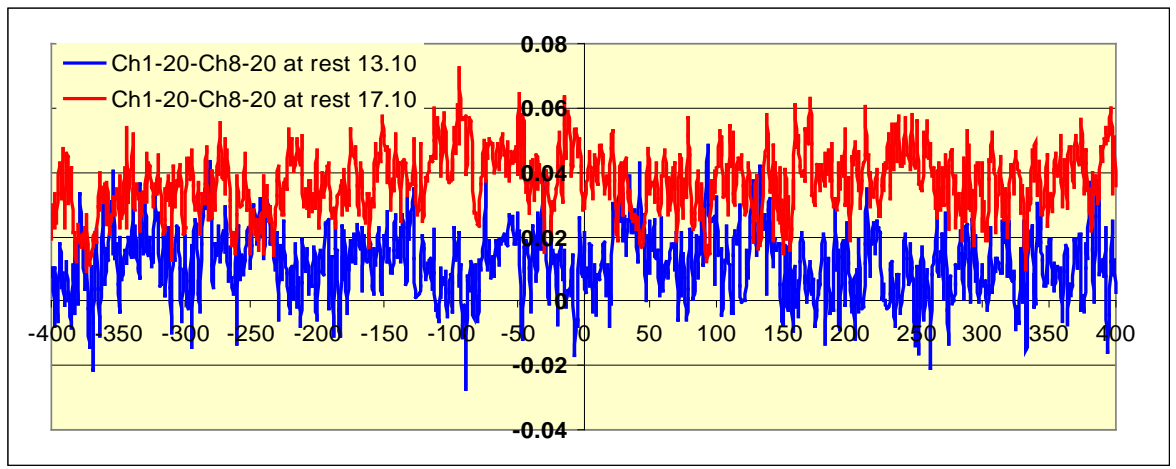

(t)

Figure 2. Examination of synchronization and coupling strength between ch8 (brain) and ch1 (right trapeze) step by step. Each figure covers a range of time of about 400 milliseconds. Each time the behaviour is given before (13.10) and after (17.10) the NPT treatment. 


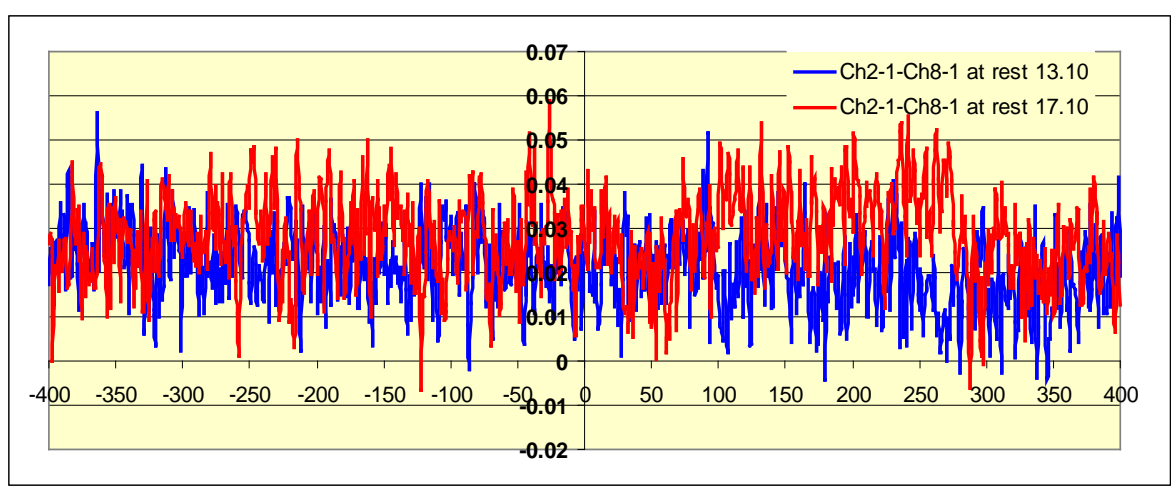

(a)

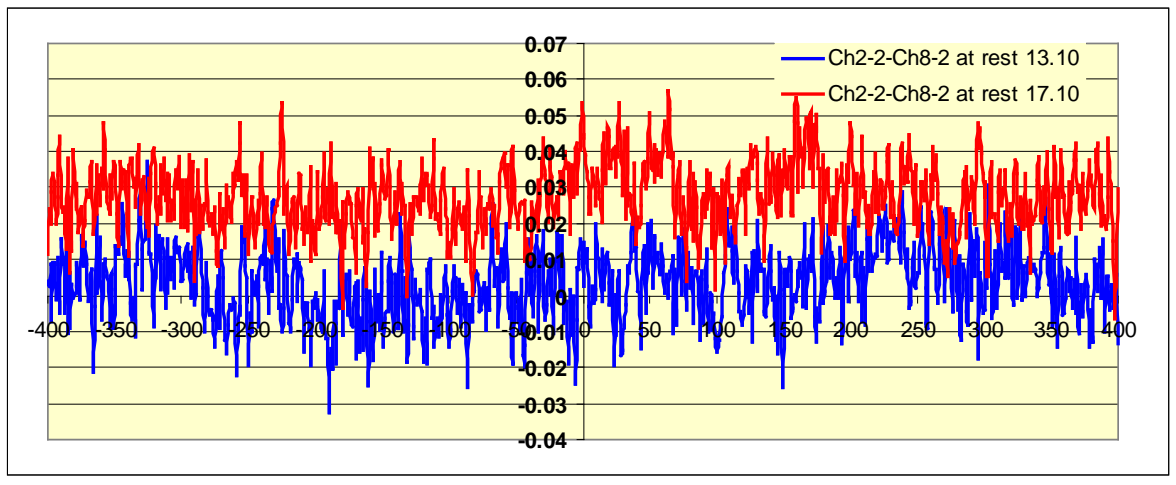

(b)

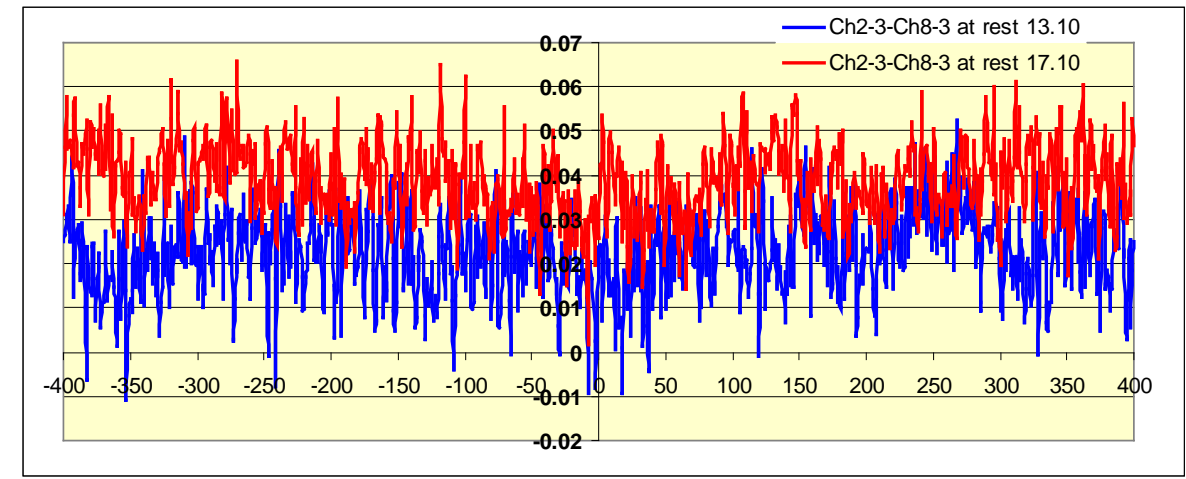

(c)

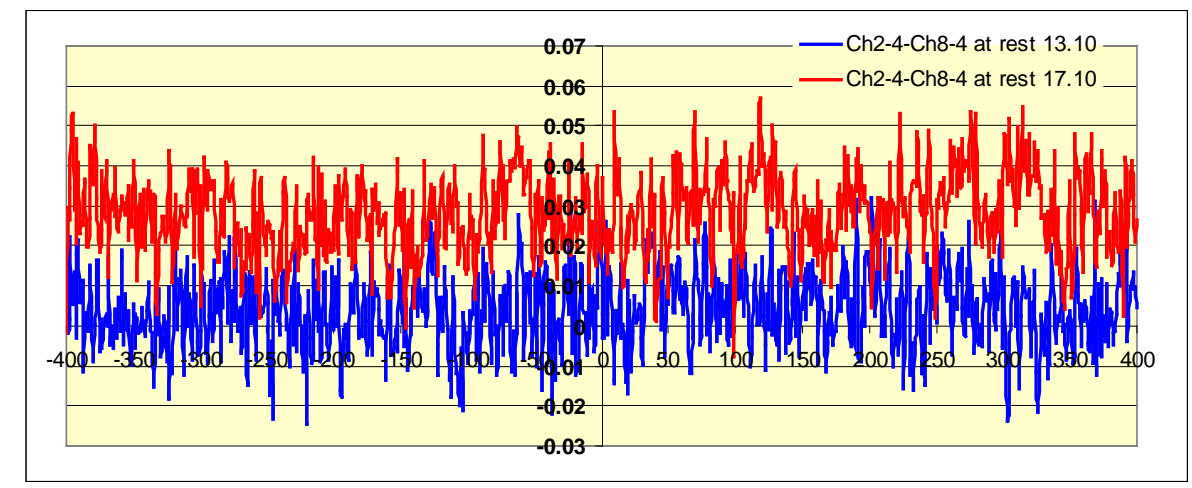

(d) 


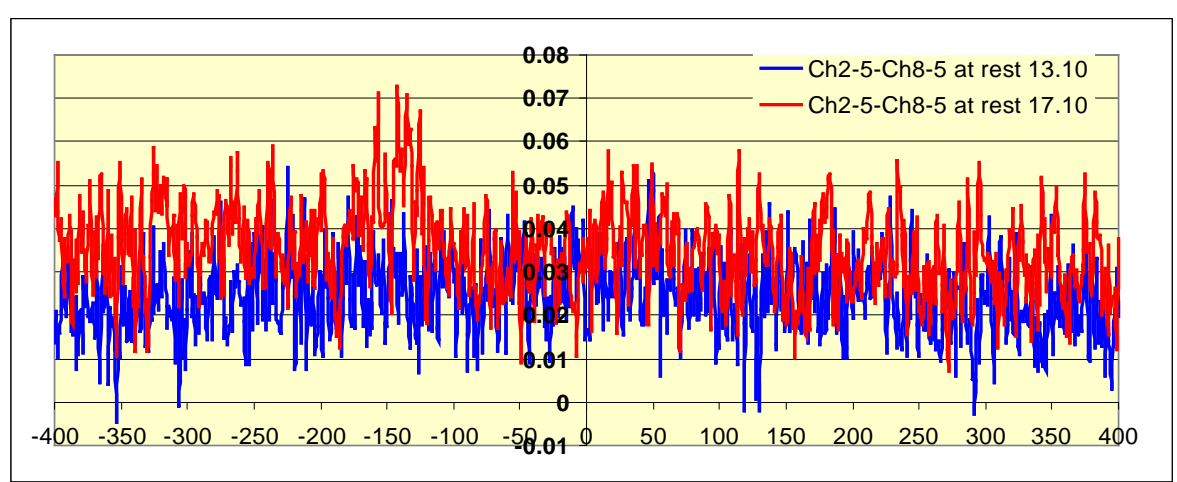

(e)

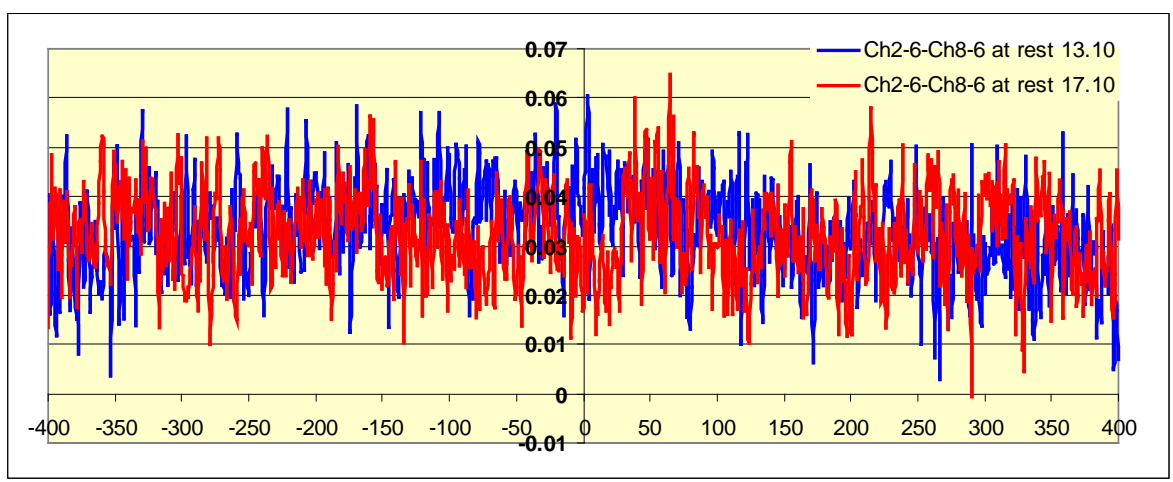

(f)

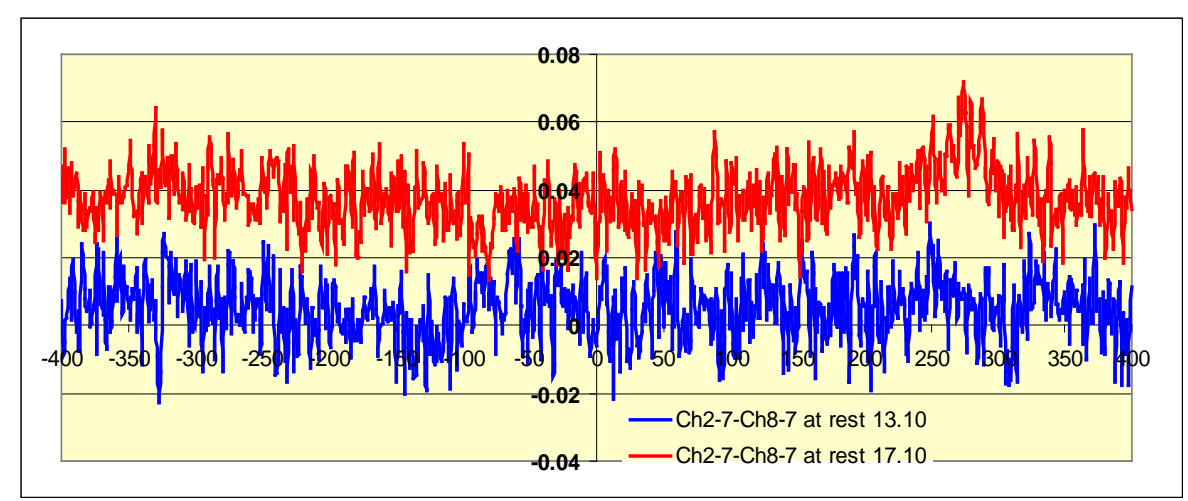

(g)

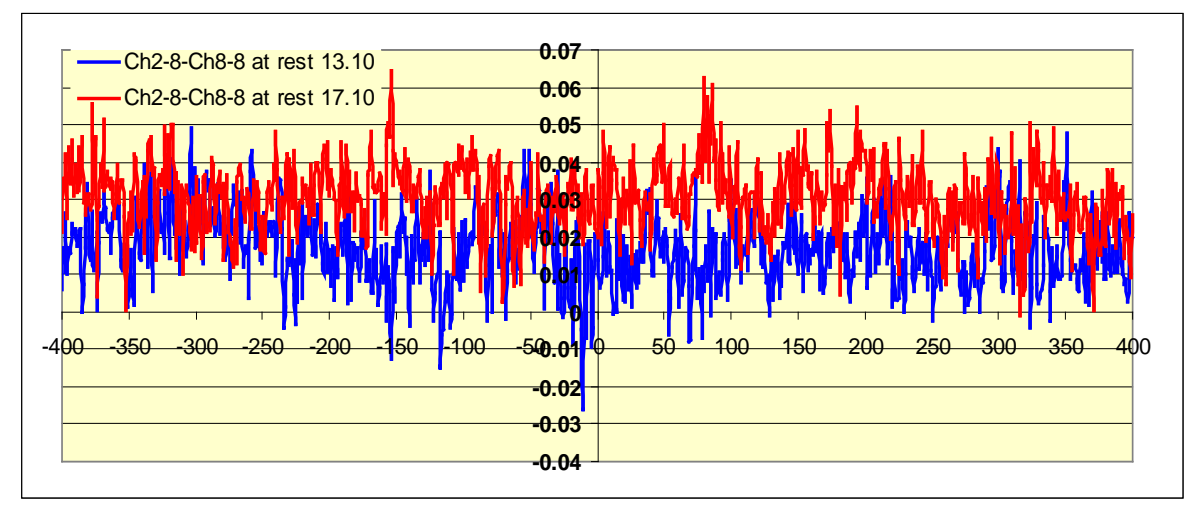

(h) 


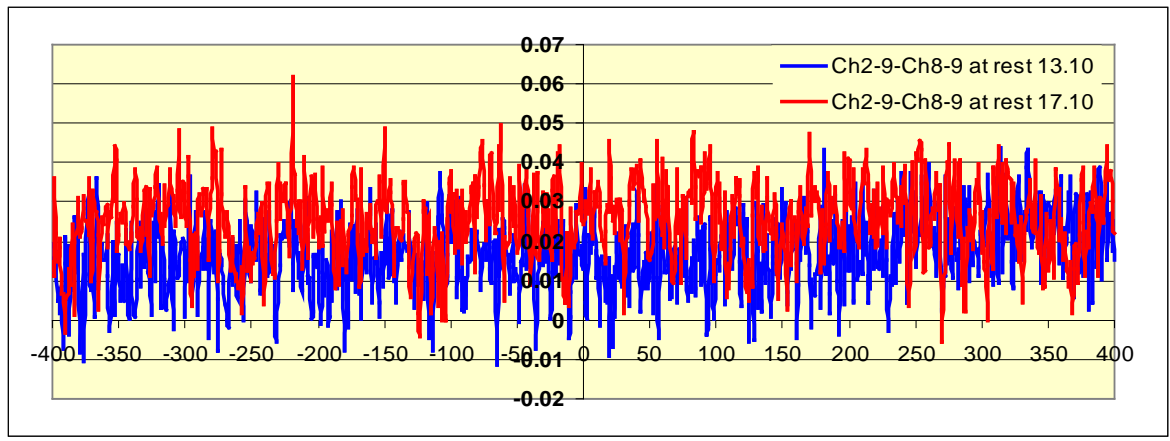

(i)

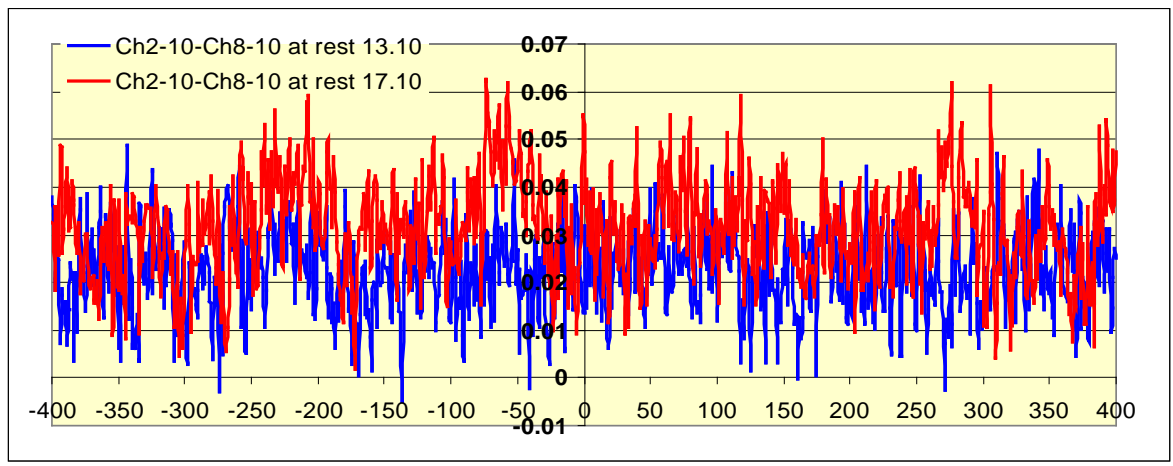

(j)

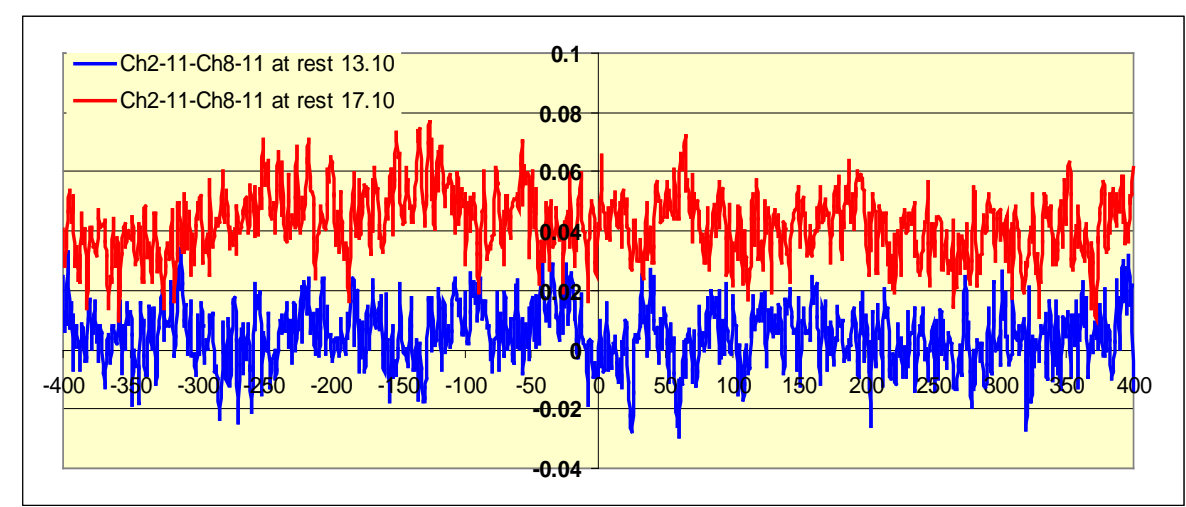

(k)

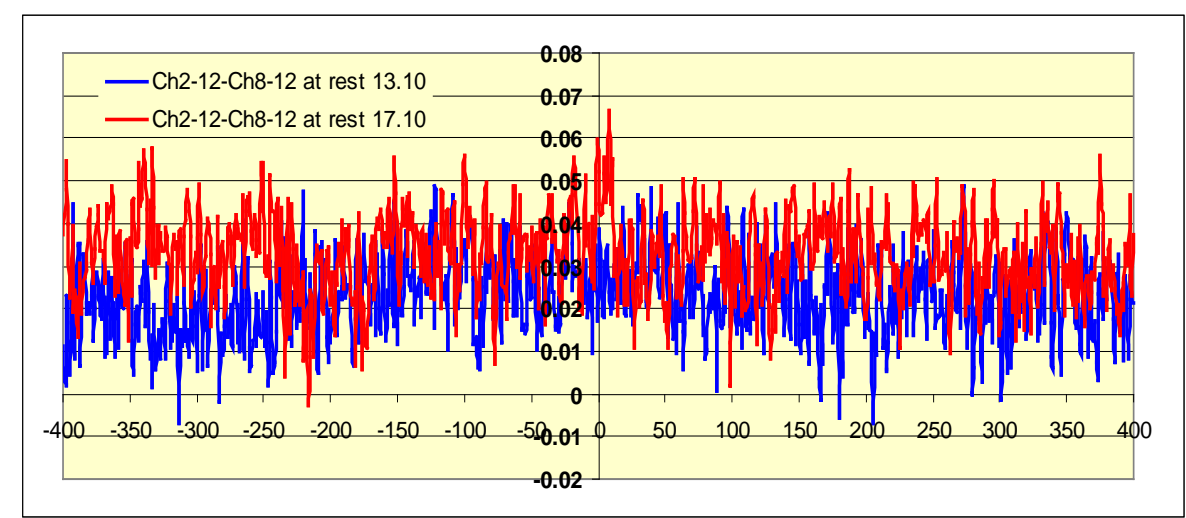




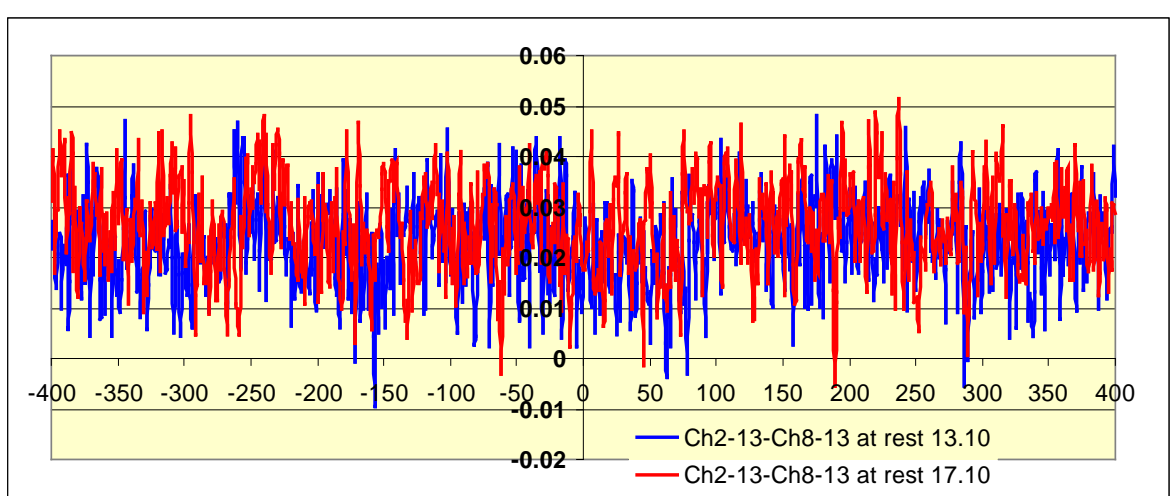

(m)

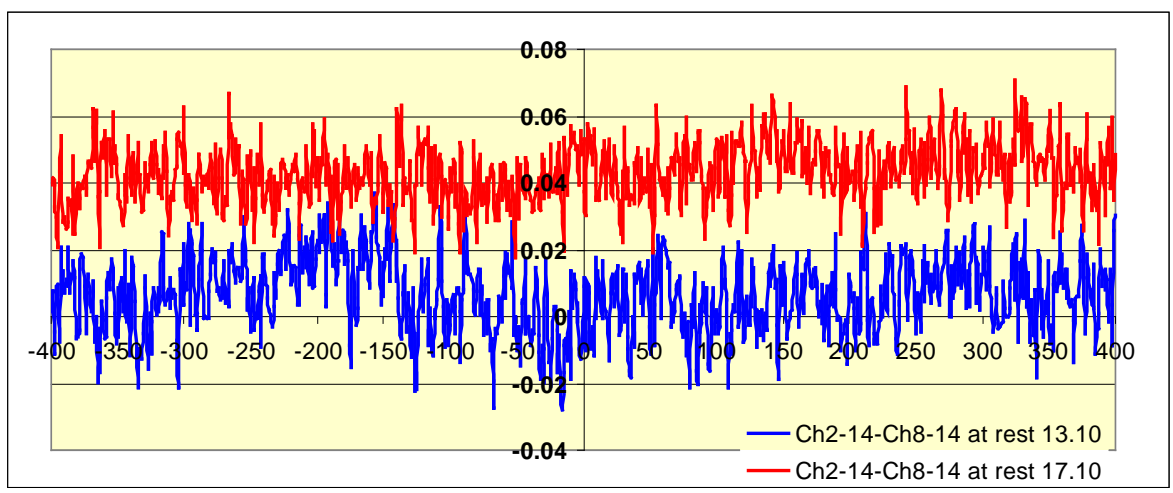

(n)

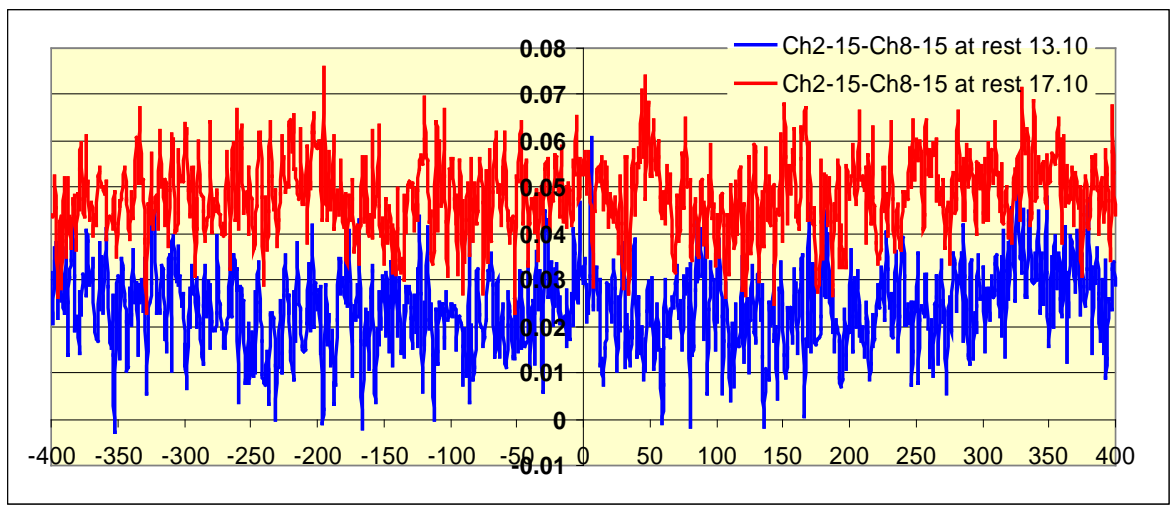

(o)

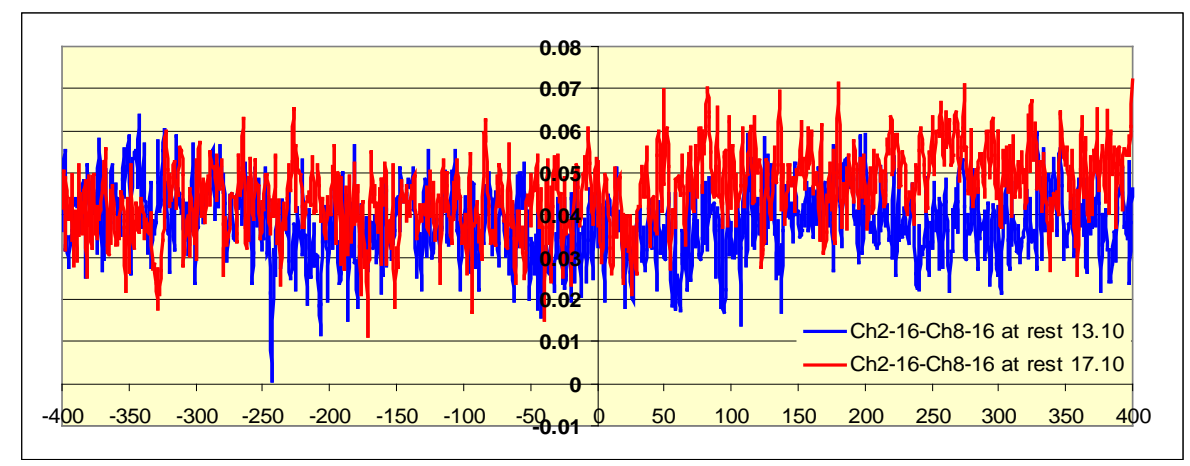

(p) 


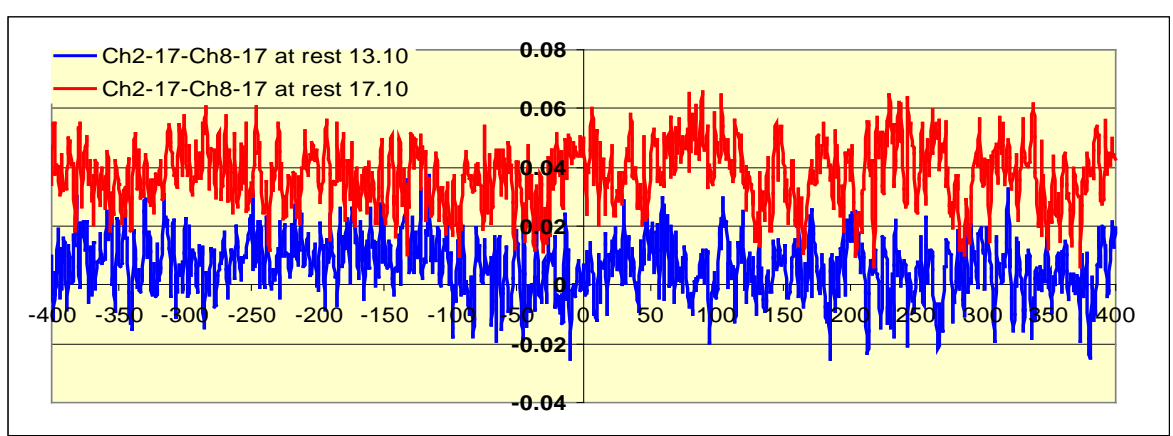

(q)

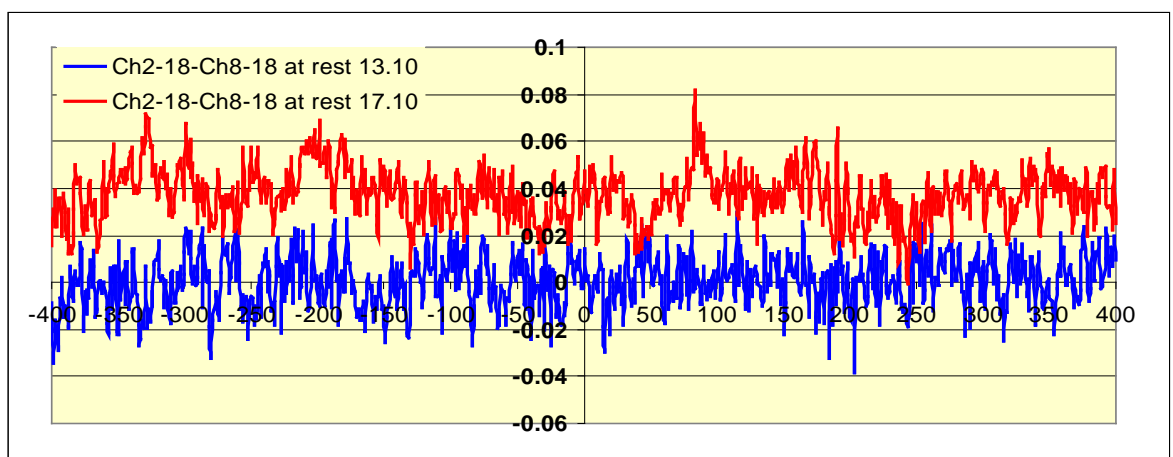

(r)

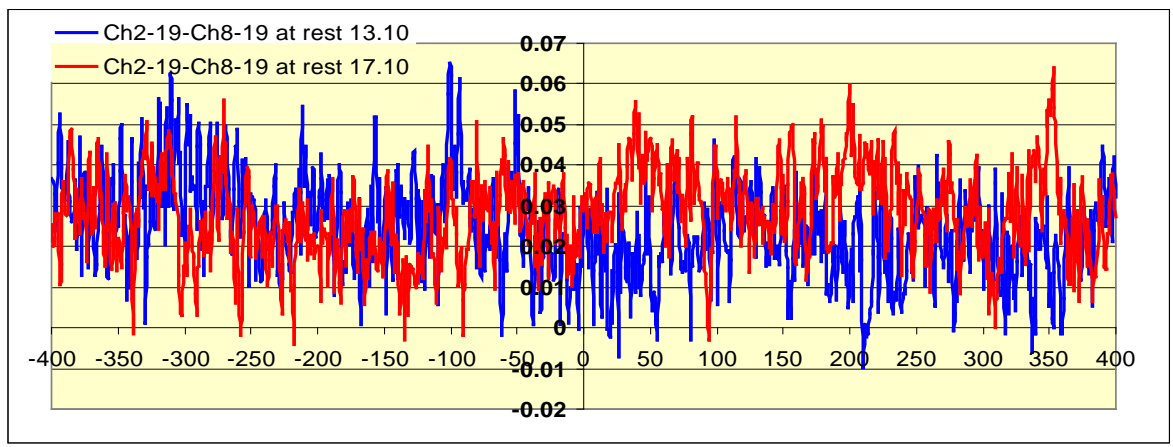

(s)

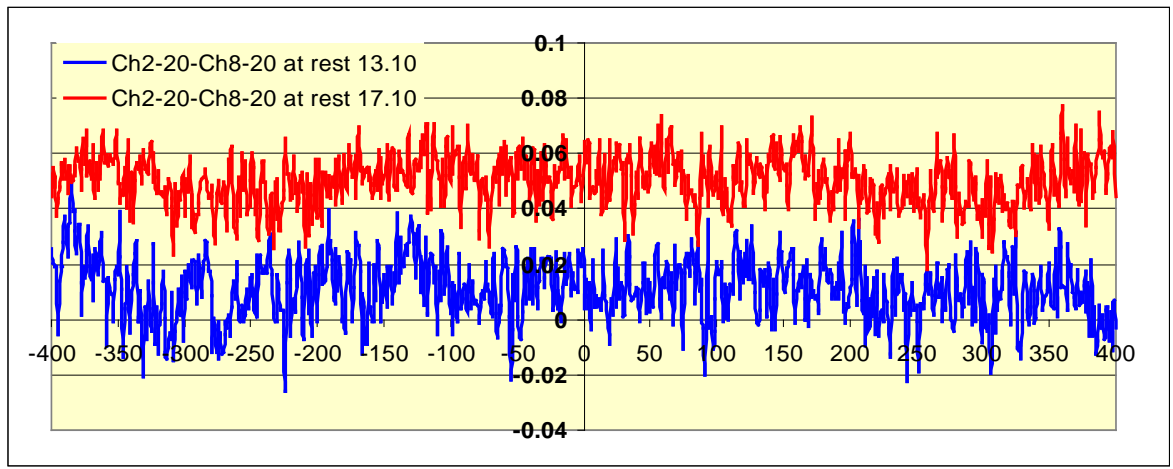

(t)

Figure 3. Examination of synchronization and coupling strength between Ch8 (brain) and ch2 (left trapeze) step by step. Each figure covers a range of time of about 400 milliseconds. Each time the behaviour is given before (13.10) and after (17.10) the NPT treatment. 
ch1-ch2 results higher before treatment respect to after treatment. In some case the difference results so relevant (from 0.16 to 0.04 ).

In Figure 1, we observe that generally we have decreased values of synchronization and coupling strength between right and left trapezes after the treatment respect to before the treatment. This is in accord with the content outlined in our previous criterium a.

This result of course indicates that right and left trapezes, following the NPT treatment, realize more dependence from Ch8 (the brain). In fact, as expected, we find the opposite situation when examining dependency and synchronization between ch1 or ch2 (trapezes) and ch8 (Brain). After the treatment, the synchronization and coupling strength increases. The results that we obtain, as well as in the case of ch1 respect to ch2, are quantified with high accuracy. Obviously, they are of valuable importance under the clinical as well as under the methodological profile. We obtain that the neurological control is higher after the performed treatment respect to the previous one before of the treatment. The results are reported in Figure 2 and Figure 3 and classify coupling strength and synchronization every 400 milliseconds as in the previous case of ch1 and ch2. All the results evidence a clear increased coupling and synchronization at each examined step.

\section{Conclusions}

The present paper concludes a cycle of research that we have performed on muscular dystrophy. After the results published in [1]-[4], in this paper we have studied the synchronization and the coupling strength between the two examined left and right trapezes and soon after between Brain and trapezes. As previously mentioned, we have used the method of the cross GMI that has enabled us to identify the peaks and the coupling strength every 400 milliseconds reaching in this manner a level of high accuracy.

It seems that we have opened a new window in the field of the methodology in the sense that we have introduced a new method that is able to explore the dynamics of the neuromuscular control in the normal as well as in the case of pathology. The values of synchronization and coupling strength are reported in ordinate in the figures and thus they are quantified at each step. Consequently, scholars may take advantage when obtaining detailed numerical values of such indexes when exploring their clinical and thus experimental conditions. The used method has exhibited a robust structure revealing its intrinsic ability to look at the inner structure of the examined signals and to account for the linear and for the nonlinear mechanisms that act to maintain neuromuscular control. It seems to us that the employed methodology represents a valuable advance at the general neurological level of analysis.

To conclude we have to comment briefly the results under the clinical profile. Such NPT treatment has evidenced that it is able to induce a net improvement. We have studied that not only muscular dystrophy but also the results relating the improvement are very encouraging. The next step is now to apply the previously described methodology to other pathological cases of interest as it is in particular the HSP (Hereditary Spastic Paraplegia). Our studies are in progress but as preliminary consideration we are in the condition to anticipate that we are obtaining still such confirming positive results.

\section{References}

[1] Ware, K., Conte, E., Marvulli, R., Ianieri, G., Megna, M., Pierangeli, E., Conte, S., Mendolicchio, L. and Pellegrino, F. (2015) Case Report: Generalized Mutual Information (GMI) Analysis of Sensory Motor Rhythm in a Subject Affected by Facioscapulohumeral Muscular Dystrophy after Ken Ware Treatment. World Journal of Neuroscience, 5, 67-81. http://dx.doi.org/10.4236/wjns.2015.52008

[2] Ware, K., Conte, E., Marvulli, R., Ianieri, G., Megna, M., Pierangeli, E., Conte, S., Mendolicchio, L. and Pellegrino, F. (2015) Analysis of the Autonomic Regulation in a Case of Facioscapulohumeral Muscular Dystrophy after Ken Ware Treatment. World Journal of Neuroscience, 5, 162-173. http://dx.doi.org/10.4236/wjns.2015.52018

[3] Conte, E., Ware, K., Marvulli, R., Ianieri, G., Megna, M., Conte, S., Mendolicchio, L. and Pierangeli, E. (2015) Chaos, Fractal and Recurrence Quantification Analysis of Surface Electromyography in Muscular Dystrophy. World Journal of Neuroscience, 5, 205-257. http://dx.doi.org/10.4236/wjns.2015.54022

[4] Conte, E., Ware, K., Marvulli, R., Ianieri, G., Megna, M., Conte, S., Mendolicchio, L. and Pierangeli, E. (2015) Heart Rate Variability: On the Importance to Perform HRV Analysis in Subjects Affected from Muscular Dystrophy. World Journal of Cardiovascular Diseases, 5, 141-149. http://dx.doi.org/10.4236/wjcd.2015.56017

[5] Pompe, B. (1993) Measuring Statistical Dependences in a Time Series. Journal of Statistical Physics, 73, 587-610. http://dx.doi.org/10.1007/BF01054341 